\title{
Projectile deformation effects on single-nucleon removal reactions
}

\author{
E. C. Simpson ${ }^{1}$ and J. A. Tostevin ${ }^{1,2}$ \\ ${ }^{1}$ Department of Physics, Faculty of Engineering and Physical Sciences, University of Surrey, Guildford, Surrey GU2 7XH, United Kingdom \\ ${ }^{2}$ National Superconducting Cyclotron Laboratory, Michigan State University, East Lansing, Michigan 48824, USA
}

(Received 20 September 2012; published 5 November 2012)

\begin{abstract}
We discuss intermediate-energy single-nucleon removal reactions from deformed projectile nuclei. The removed nucleon is assumed to originate from a given Nilsson model single-particle state and the inclusive cross sections, to all rotational states of the residual nucleus, are calculated. We investigate the sensitivity of both the stripping cross sections and their momentum distributions to the assumed size of the model space in the Nilsson model calculations and to the shape of the projectile and residue. We show that the cross sections for small deformations follow the decomposition of the Nilsson state in a spherical basis. In the case of large and prolate projectile deformations the removal cross sections from prolate-like Nilsson states, having large values for the asymptotic quantum number $n_{z}$, are reduced. For oblate-like Nilsson states, with small $n_{z}$, the removal cross sections are increased. Whatever the deformation, the residue momentum distributions are found to remain robustly characteristic of the orbital angular momentum decomposition of the initial state of the nucleon in the projectile.
\end{abstract}

DOI: 10.1103/PhysRevC.86.054603

PACS number(s): 24.50.+g, 25.70.Mn, 25.60.Je, 21.10.Pc

\section{INTRODUCTION}

Radioactive beam facilities are giving access to a wealth of highly exotic isotopes, many produced for the first time using fast reactions induced by low-intensity intermediate-energy secondary beams. Significant deviations from the pattern of shell structure found in stable isotopes have been observed, with growing evidence for the quenching of shell gaps and of the breakdown of the conventional neutron magic numbers in very neutron-rich nuclei. Intense experimental effort has been focused on the island of inversion, originally identified in mass measurements of the neutron-rich sodium isotopes [1], where the quenching of the $N=20$ shell gap near ${ }^{31} \mathrm{Na}$ leads to strong $f p$-shell intruder configurations in the ground-state wave functions. Studies of $N=28$ isotones also show progressively decreasing first $2^{+}$-state energies, evidence of configuration coexistence in ${ }^{44} \mathrm{~S}$ [2,3], and gap quenching in ${ }^{42} \mathrm{Si}$ [4]. Density functional calculations also predict alternating prolate and oblate ground states along the $N=28$ isotone chain [5]. There is also evidence that the $N=40$ subshell closure is quenched near ${ }^{64} \mathrm{Cr}$, deduced from the systematics of intermediate-energy inelastic scattering cross sections [6], two-proton removal reactions [7], and ground-state to first $2^{+}$-state $B(E 2)$ values in ${ }^{62-66} \mathrm{Fe}[8,9]$.

An effective tool for studying the near-Fermi-surface structure of exotic nuclei is to use fast nucleon removal reactions [10], where one or two nucleons are removed from an exotic projectile in an intermediate energy (typically $>80 \mathrm{MeV}$ /nucleon) surface-grazing collision with a beryllium or carbon target. To date the experimental observations made were largely inclusive with respect to the final states of the target and the removed nucleons and so are limited to the properties of the heavy reaction residues. Specifically, data observe (a) the possible decay $\gamma$ rays from excited residue final states, (b) absolute cross sections, and (c) the distribution of the cross sections with the (usually beam-directional) momenta of the reaction residues. Depending on the beam intensity, the reaction, the final-state populations, and the detection capabilities, (b) and/or (c) may be inclusive or exclusive in the populated final states of the residual nuclei.

The high experimental efficiency of the intermediate energy knockout technique has facilitated studies on some of the most exotic nuclei, produced as low-intensity secondary fragmentation beams. Access to nuclei far from the valley of $\beta$ stability has helped identify regions of rapidly changing nuclear structure. Early studies using single-nucleon removal focused on halo nuclei, where narrow residue momentum distributions were observed, the result of very weak nucleon binding in states with low orbital angular momentum. These results were successfully interpreted using models where the target was assumed to act as a black, absorptive disk [11,12]. In general, the widths of measured residue momentum distributions are characteristic of the orbital angular momentum, $\ell$, of the spherical single-particle orbital occupied by the removed nucleon, with larger $\ell$ leading to wider distributions. The removal cross sections decrease with increasing nucleon binding energy and $\ell$, reflecting the reduced spatial extension of the nucleon's wave function relative to the projectile core owing to its greater binding and its increased centrifugal barrier, respectively.

For single-nucleon removal, under the assumption that the residual nucleus behaves as a spectator whose state is not coupled to the dynamics of the collision, the theoretical cross sections factor into structural and reaction dynamical terms. The nuclear structure input comes in the form of the single-nucleon overlap function and its normalization, the spectroscopic factor. The reaction dynamics information is encapsulated in the single-particle cross section, depending sensitively on the relative sizes of the projectile core and the single-nucleon wave function, with the latter influenced, as stated above, by the nucleon separation energy and its $\ell$ value. Experimental studies are becoming more sophisticated with 
measurements of the final states of the removed nucleons also now possible for both one- and two-nucleon removal $[13,14]$. While not yet routinely possible, such more-exclusive analyses have provided essential tests of the assumed reaction mechanisms and their contributions to the removal cross sections.

A body of experimental data on the removal of nucleons of a deficient species from highly asymmetric nuclei, that is, the removal of nucleons whose Fermi-surface is now well bound, has revealed knockout cross sections to be suppressed compared to the eikonal plus shell-model removal reaction predictions. These suppressions are conveniently characterized as having small values of the ratio $R_{s}=\sigma_{\exp } / \sigma_{\text {th }}$, where the theoretical cross sections $\sigma_{\text {th }}$ incorporate the collision dynamics and structure input from spherical, truncated-basis shell-model form factors and their spectroscopic factors $[15,16]$. These suppressions, in part owing to the short and longer-ranged nucleon correlations identified in stable nuclei and quantified using $\left(e, e^{\prime} p\right)$ proton-removal systematics (e.g., Ref. [17]), may be supplemented by effects owing to the proximity and coupling of nucleons of the excess species to the continuum [18]. However, there is also evidence for deformation in many of these asymmetric $s d$-shell systems. These ground-state deformations are not known in the most exotic cases but for ${ }^{34} \operatorname{Ar}(-1 \mathrm{n})$, where $R_{s}=0.41 \pm 0.07$ was found, the deformation derived from the ground-state to first $2^{+}$-state $B(E 2)$ value is $\beta\left({ }^{34} \mathrm{Ar}\right)=0.44 \pm 0.07$ [19]. The effects of deformation are thus of importance.

The Nilsson model has been used extensively to study single-particle degrees of freedom in deformed nuclei [20]. In its simplest form the Nilsson model generates nonspherical single-particle states of a deformed harmonic oscillator potential, supplemented by spin-orbit and angular momentum terms in its Hamiltonian. For nonzero deformation the nucleon states no longer have good $j$ but are an admixture of the contributing spherical basis states $(n \ell j)$, an admixture that evolves with the deformation $\delta$. Single-nucleon transfer reactions have often been used to identify the fingerprints of deformation and the associated Nilsson states in nuclei, the relative cross sections to different final states of a given rotational band being characteristic of their components in a spherical-basis structure [21], allowing the assignment of rotational bands to particular Nilsson configurations.

The currently used (eikonal) theoretical reaction models for nucleon removal reactions are rooted in optical interactions and structure amplitudes from truncated-basis (shell) models with spherical bases. In many cases this may be a poor assumption. The effects of deformation will impact both the structural and the reaction dynamical aspects. The sampling of the single-nucleon overlap by the target is determined by the sizes of the target and projectile core. With a deformed projectile the orientation of the projectile intrinsic symmetry axis with respect to the target will alter the geometry and the volume probed with possible effects on both the magnitudes of cross sections and the residue momentum distributions arising from nucleon removal. Total reaction cross sections for deformed projectiles are expected to show a significant sensitivity to the orientation of the projectile [22], originating from the orientation dependence of the highly absorptive projectile-target interaction [23]. Recent systematic studies have emphasized the role of deformation in enhancing reaction cross sections, with implications for the underlying single-particle structure near the island of inversion [24,25]. Furthermore, new tests of deformed structure models are desirable.

Very few attempts have been made to incorporate deformation into nucleon removal reactions. Sakharuk and Zelevinsky [26] also studied the single-nucleon stripping term in a strong coupling model, using Nilsson states coupled to a deformed core. Two models were presented, one using Glauber-type deformed potential $S$ matrices [27], and thus incorporating dynamic excitations of the residue during its interaction with the target, and a simpler geometric model based on the absorptive radius (black-disk) approximation. Their results suggested that early data for ${ }^{25} \mathrm{Al}(-1 \mathrm{p})$ [28], whose momentum distribution showed a hint of having shoulders, could be explained as resulting from dynamic excitations of different states of the residue. A weak dependence of the magnitudes of the stripping cross sections on the degree of deformation was found. The residue momentum distributions were found to be indicative of the orbital angular momentum content of the Nilsson state. Deformation effects on elastic breakup, of importance for weakly bound deformed-core systems, were also considered, in a weak-coupling model [29], assuming spherical single-particle states coupled to a deformed core. The calculated stripping cross sections were once again found to be weakly dependent on the assumed core deformation.

In this paper we present a further more systematic assessment of the sensitivities of nucleon removal cross sections and momentum distributions to deformation. We adopt the strong-coupling limit, applicable to the removal of well-bound nucleons, and assume the removed nucleon to originate from a Nilsson state $v$ with angular momentum projection $m$ on the projectile intrinsic symmetry axis, $\hat{\Omega}$. We assume also that the highly absorptive interaction of the projectile core (the residual nucleus), treated as a quadrupole-deformed spheroid, with the spherical target, and their associated elastic $S$ matrix, may be approximated using a sharp-cutoff, strong absorption model. These two approximations, namely (a) the use of harmonic oscillator-based (Nilsson) wave functions, having the wrong radial asymptotic form, and (b) the use of the absorptive-disk model, are thus two inherent limitations of the model used. We quantify their expected effects on the magnitudes of the calculated absolute cross sections; however, these limitations have no consequences for the conclusions we draw as to the fractional changes in cross sections and the qualitative trends of the effects of deformation. Here we study systematically these sensitivities of cross sections and momentum distributions, for Nilsson states from the $N=2(s d-)$ shell, making clear the observed phenomena in terms of underlying changes to the Nilsson states. In Sec. II we outline the notation used for the single-particle orbitals in deformed nuclei using the Nilsson model, highlighting those aspects relevant to nucleon removal reactions. In Sec. III we then describe the nucleon removal model used and in Sec. IV present a systematic study of nucleon removal outcomes, using mass $A=28$ as a generic $s d$-shell example. 


\section{DEFORMED SINGLE-PARTICLE STATES: THE NILSSON MODEL}

The Nilsson model, which gives the single-particle energies of nucleons moving in a deformed modified harmonic oscillator potential [20], has been highly successful in (a) explaining the general features of deformed nuclei and (b) identifying single-particle structures and their corresponding rotational bands, including in light nuclei [30-34]. Singlenucleon transfer reaction studies have often provided the probe of such deformed single-particle and Nilsson model wave functions, where cross sections to the states of a rotational band follow patterns that are characteristic of an underlying Nilsson model configuration [21]. We review very briefly the Nilsson model, particularly those aspects pertinent to the present discussions.

The Nilsson model Hamiltonian uses a deformed oscillator potential with added spin-orbit and centrifugal terms,

$$
\begin{aligned}
H= & -\frac{\hbar^{2}}{2 M} \nabla^{2}+\frac{M}{2}\left[\omega_{\perp}^{2}\left(x^{2}+y^{2}\right)+\omega_{z}^{2} z^{2}\right] \\
& -2 \hbar \stackrel{\circ}{0}_{0} \kappa \vec{\ell} \cdot \vec{s}-\hbar \stackrel{\circ}{0}_{0} \mu \kappa\left[\ell^{2}-\left\langle\ell^{2}\right\rangle_{N}\right],
\end{aligned}
$$

where $M$ is the nucleon mass. In the final term $\left\langle\ell^{2}\right\rangle_{N}$ is the average of $\ell^{2}$ for a given oscillator shell $N$ and its presence maintains the appropriate spacing of $\hbar \omega_{0}$ between major shells. The deformation of the oscillator potential can be defined in terms of the parameter $\delta$, related to the oscillator frequencies by

$$
\omega_{\perp}^{2}=\omega_{0}(\delta)^{2}\left(1+\frac{2}{3} \delta\right), \quad \omega_{z}^{2}=\omega_{0}(\delta)^{2}\left(1-\frac{4}{3} \delta\right),
$$

where volume conservation of the deformed oscillator implies a dependence of $\omega_{0}(\delta)$ on the deformation, that is,

$$
\omega_{0}(\delta)=\stackrel{\circ}{\omega}_{0}\left(1-\frac{4}{3} \delta^{2}-\frac{16}{27} \delta^{3}\right)^{-1 / 6} .
$$

Tables of the single-particle solutions of this Nilsson Hamiltonian can be found in the literature (e.g., Refs. $[20,35,36]$ ). The shape of the quadrupole-deformed equipotential surface is often parametrized using

$$
R(\theta, \phi)=R_{c}\left[1+\beta Y_{20}(\theta, \phi)\right],
$$

where $(\theta, \phi)$ refer to the nuclear symmetry axis $\hat{\Omega}$. To first order, the two deformation parameters $\delta$ and $\beta$ are related by

$$
\beta \approx \frac{2}{3} \sqrt{\frac{4 \pi}{5}} \delta=1.057 \delta
$$

Solutions of the Nilsson model often utilize stretched coordinates, denoted by a deformation parameter $\epsilon$ [20], in which a coordinate transformation is made that, for a pure quadrupole deformation, eliminates exactly the couplings between different major shells. This reduces very significantly the computational effort in the matrix diagonalization. The disadvantage of this approach for the present discussion is that the coordinate transformation is different in the $z$ and the perpendicular directions. Because we require the single-nucleon wave functions in the laboratory (unstretched) coordinates, the use of stretched coordinates in the Nilsson calculation necessitates a subsequent expansion in the unstretched coordinates, and that reintroduces the couplings.
Instead, we perform the Nilsson calculations in the unstretched coordinates, parametrized by deformation $\delta$, and take account of the couplings between different major shells (see also Ref. [37]). This no longer presents a significant computational challenge. In principle, the resulting wave functions are independent of the coordinates used, provided the potential is the same. However, there is no definite prescription for generalizing the spin-orbit and angular momentum terms in the Hamiltonian when making the coordinate change [38]; the stretched coordinates refer only to the deformed oscillator itself. Turning off the $\vec{\ell} \cdot \vec{s}$ and $\ell^{2}$ terms allowed us to verify that the large-basis diagonalization and wave-function stretching procedure give consistent results.

The Nilsson states themselves have good projection, $m$, of angular momentum on the intrinsic symmetry axis $\hat{\Omega}$ and, for the potentials with axial-symmetry considered here, are doubly degenerate $( \pm m)$. For large values of the deformation the states of the Nilsson potential tend to pure cylindrical oscillator states and are then often labeled by the asymptotic quantum number set $\left[N n_{z} \lambda\right] m$, but in general have neither good cylindrical nor spherical $(n \ell j)$ quantum number sets.

Our primary emphasis here will be of nucleon removal from $s d$-shell isotopes. The appropriate Nilsson model levels diagram for $N=2$ is shown in Fig. 1. The spherical quantum numbers of the states are indicated at zero deformation and the asymptotic labels $\left[\mathrm{Nn}_{z} \lambda\right] \mathrm{m}$ are shown on the right-hand side of the plot. For prolate deformations the states with large values of the cylindrical oscillator number $n_{z}$, the number of $z$-directional quanta, decrease in energy and conversely for the states with smaller $n_{z}$. In general, for a given $\delta$, each Nilsson orbit is a mixture of the spherical states with different total $j$ and orbital $\ell$ angular momenta, with the degree of mixing dependent on $\delta$ and the state's projection $m$. One can expand

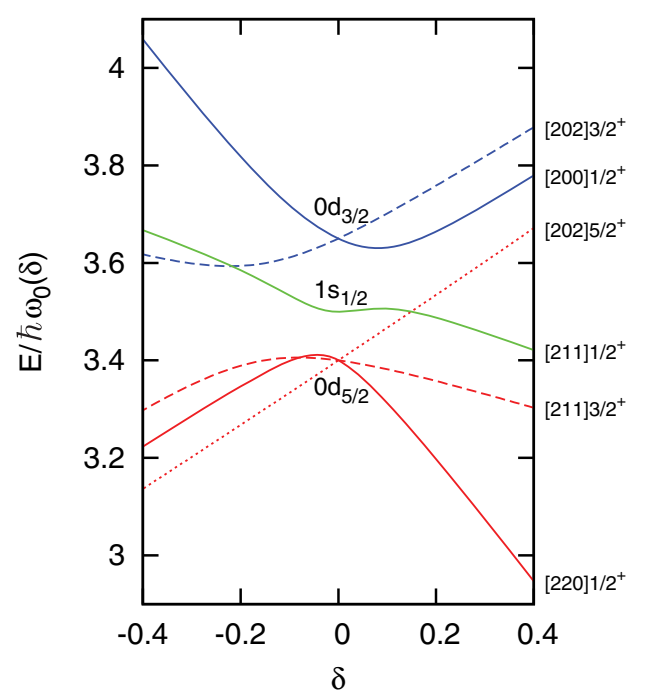

FIG. 1. (Color online) Nilsson model calculations for nucleons in the $s d$ shell assuming a pure $N=2$ model space and the parameters $\kappa=0.05$ and $\mu=0.0$ of Ref. [35]. The plot shows states with $m=$ $1 / 2$ (solid lines), $3 / 2$ (dashed lines), and 5/2 (dotted lines), colored by the originating spherical state for zero deformation (red, $0 d_{5 / 2}$; blue, $0 d_{3 / 2}$; green, $1 s_{1 / 2}$ ). The asymptotic quantum numbers $\left[N n_{z} \lambda\right]$ for each state are indicated on the right-hand edge of the plot. 
the Nilsson states $\nu m$ in either a spherical basis $(n \ell j)$ as

$$
|v m\rangle=\sum_{n \ell j} a_{v m}^{n \ell j}|n \ell j m\rangle,
$$

or in a cylindrically symmetric oscillator (asymptotic) basis, as

$$
|v m\rangle=\sum_{n_{z} n_{s} \lambda} a_{v m}^{n_{z} n_{s} \lambda}\left|n_{z} n_{s} \lambda\right\rangle .
$$

The quantum numbers of the cylindrical oscillator states are such that $N=n_{z}+2 n_{s}+\lambda$.

With $N=2$, the $s d$ shell, only Nilsson states with $m=1 / 2$ can mix $s$ - and $d$-state components. Those with $m=3 / 2$ and $5 / 2$ mix only the $d$-wave components, while the [202]5/2 state remains purely $0 d_{5 / 2}$, independent of the deformation. Figure 2 shows these squared spherical component amplitudes $\left[a_{v m}^{n \ell j}\right]^{2}$ as a function of the assumed deformation for the three $m=1 / 2$ Nilsson states within a pure $N=2$ model space. Because the residue momentum distributions of removal reactions from spherical nuclei have widths that are characteristic of the orbital angular momentum of the removed nucleon, we anticipate sensitivity in this observable to these changes in the $\ell$ composition of the Nilsson states as a function of deformation.

Mixing of the $N=2$ states with orbitals in higher and lower major shells, that is, with $|\Delta N|=2$, will also mix different $\ell$ components into all of the states, regardless of $m$, though

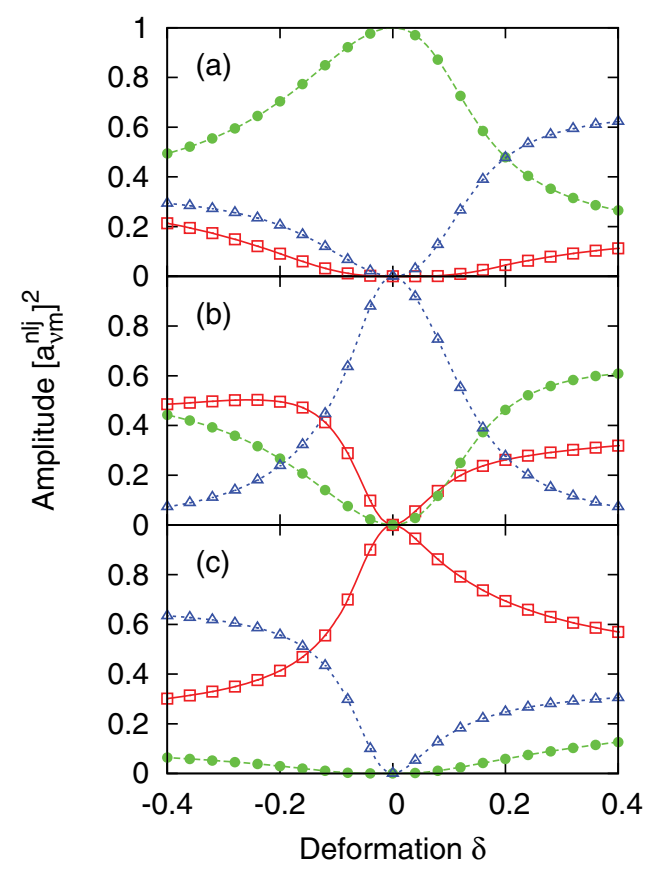

FIG. 2. (Color online) Squared amplitudes $\left[a_{v m}^{n \ell j}\right]^{2}$ for the expansion of the Nilsson state $(v 1 / 2)$ in a spherical basis $n \ell j$. The panels show the results for states with asymptotic quantum numbers (a) $[200] 1 / 2$, (b) $[211] 1 / 2$, and (c) [220]1/2 and decrease in energy from top to bottom. The lines show the amplitudes of the $0 d_{5 / 2}$ (red solid lines, open squares), $0 d_{3 / 2}$ (green long-dashed lines, solid circles), and $1 s_{1 / 2}$ (blue short-dashed lines, open triangles) spherical components in each case. these components will be considerably smaller than those from the mixing within the $N=2$ shell. Below we assess the significance of these smaller $\Delta N= \pm 2$ contributions to wave functions and to the knockout cross sections by using different numbers of oscillator shells in the Nilsson model calculations. This is described and quantified in the next section.

\section{ABSORPTIVE-DISK MODEL FOR NUCLEON REMOVAL}

At intermediate energies the nucleus-nucleus optical interactions between the beryllium (or carbon) target nuclei and the $s d$-shell projectile core nuclei are very highly absorptive. This strong absorption is reflected in the vanishing of the core-target elastic $S$ matrix, $\left|S_{c}\left(b_{c}\right)\right|^{2}=0$, for smaller than surface-grazing impact parameters, $b_{c}$, of the pair. This strong absorption essentially determines those $b_{c}$ values for which the core nucleus may survive the collision and so contribute to the nucleon-removal reaction yield. The additional requirement that a (well-bound) valence nucleon near the Fermi surface of the projectile is both present and also interacts strongly (and inelastically) with the target nucleus then severely restricts the projectile-target impact parameters for which the nucleon removal takes place. Typically, the eikonal approximation is used to construct the core- and nucleon-target $S$ matrices from their optical potentials; that is, these fast fragments are assumed to follow straight-line paths during their short interaction times and small strong interaction distances with the (light) target nuclei. We exploit these ideas for singlenucleon removal from deformed nuclei.

We assume the projectile consists of a single particle in a given Nilsson state, $v m$, bound to an axially symmetric deformed core. A schematic of the direct reaction mechanism is shown in Fig. 3. In each collision the spherical target will probe a cylindrical volume bored through the single-particle wave function, sampling its Nilsson state. The core of the projectile is assumed to be a uniform solid spheroid and its impact parameters $b_{c}$ must be sufficiently distant from the target that the pair do not overlap at any point on their passing trajectories along the $z$ direction, or else $\left|S_{c}\left(b_{c}\right)\right|^{2}=0$. Their minimum impact parameter, $b_{\min }$, is therefore dependent on the

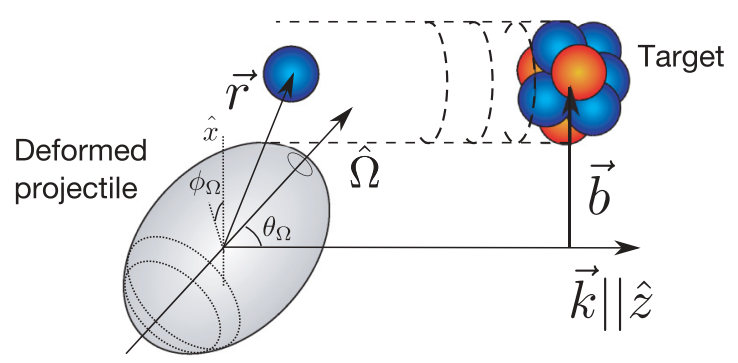

FIG. 3. (Color online) Schematic of the deformed projectile (left) incident on the absorptive target (right). The target nucleus samples a volume of the projectile near the surface. The interaction of the projectile and target and the volume of the projectile probed will depend on the orientation of the projectile relative to the laboratory $z$ axis, denoted by $\hat{\Omega} \equiv\left(\theta_{\Omega}, \phi_{\Omega}\right)$, and its deformation $\delta$. 
degree of deformation $\delta$ and the orientation $\hat{\Omega}$ of the projectile core with respect to the beam direction, $\hat{z}$. Thus, how the valence nucleon wave function is sampled depends on both its state and its orientation. The cross sections calculated below are inclusive with respect to states of the rotational band of the deformed core.

The added complexity, when extending the methods to deformed nuclei, is thus primarily computational, because the cross section for each projectile orientation must now be computed and averaged over. Our approach here is (a) to expand the intrinsic state wave function in a cylindrical oscillator basis of the laboratory system, and (b) to use a simplifying (absorptive-disk) approximation for the core-target interaction. These formal manipulations of the valence nucleon wave function are described in the next section and allow all beam-directional integrals to be evaluated analytically. Conventional models for nucleon removal from spherical projectiles use folding models and the nuclear densities to compute the projectile-constituent-target elastic $S$ matrices. We use a hybrid approach and assume the nucleus-nucleus, core-target $S$ matrix is well described using a sharp-cutoff $S$ matrix. For the nucleon-target interaction, which is responsible for the nucleon removal and describes the degree of inelasticity in the nucleon-target system, we retain the single-folding model approach based on an assumed target density.

Based on calculations for spherical nuclei the absorptivedisk model is expected to calculate absolute nucleon removal cross sections with an accuracy of order $30 \%$, producing smaller cross sections. These are sufficient to understand the essential features of deformation. Further, our calculations account for only the stripping mechanism; however, because we are interested in well-bound (strongly coupled) deformed systems, the elastic breakup terms are less important. For spherical nuclei and separation energies such as used here, typically $20-30 \%$ of nucleon removal events involve diffractive (elastic) breakup. Our main aim here is to investigate the sensitivity of cross sections and their distribution with the residue momenta to deformation.

In the following sections we discuss the inputs into the model, beginning with a discussion of the shapes of the projectile core (the residue), the Nilsson model wave functions used, and the calculation of the cross section itself. We then discuss the limit of small deformations and general qualitative expectations of the model. A specific application of the model to mass $A=28$ projectiles is then presented in Sec. IV, accompanied by comparative spherical model calculations.

\section{A. Wave functions}

We first discuss the Nilsson single-nucleon wave functions and their practical use in the knockout calculations. Denoting the Nilsson wave function of the valence nucleon $v m$ then in the projectile intrinsic frame we first expand it in a spherical basis denoted by the quantum numbers $(n \ell j)$

$$
\Psi_{v m}(\vec{r}, \hat{\Omega})=\sum_{n \ell j} a_{v m}^{n \ell j} \Psi_{n \ell j m}(\vec{r}, \hat{\Omega}),
$$

with coefficients $a_{v m}^{n \ell j}$, obtained from the Nilsson model calculations. We have included explicitly the notation $\hat{\Omega}$ to denote the dependence on the symmetry axis of the projectile, the axis for which the projection of the angular momentum is $m$. The polar angles of $\hat{\Omega}$ define the orientation of the symmetry axis relative to the laboratory $\hat{z}$ axis. In principle, the wave function is free to mix contributions from different major oscillator shells $N$, though the degree to which this occurs is deformation dependent.

We now use the rotational properties of the spherical wavefunction components to express the Nilsson wave function in the spherical basis with projections $m^{\prime}$ on the laboratory $z$ axis; that is,

$$
\Psi_{v m}(\vec{r}, \hat{\Omega})=\sum_{n \ell j} a_{v m}^{n \ell j} \sum_{m^{\prime}} \mathcal{D}_{m^{\prime} m}^{j}(\hat{\Omega}) \Psi_{n \ell j m^{\prime}}(\vec{r}, \hat{z}) .
$$

The spherical wave functions are, in conventional notation,

$$
\Psi_{n \ell j m}(\vec{r}, \hat{z})=\sum_{\lambda \sigma}(\ell \lambda s \sigma \mid j m) \psi_{n \ell \lambda}(\vec{r}, \hat{z}) \chi_{s \sigma},
$$

where the spherical oscillator model wave functions are

$$
\psi_{n \ell \lambda}(\vec{r}, \hat{z})=u_{n \ell}(r) Y_{\ell \lambda}(\hat{r}),
$$

with the usual forms for the radial parts $u_{n \ell}(r)$. The phases were chosen such that the $u_{n \ell}(r)$ were positive near the origin and no $i^{\ell}$ factors were present. These radial oscillator wave functions do not depend on $j$.

Finally, for reasons of computational efficiency within the eikonal reaction model, with its beam-direction cylindrical symmetry, it is useful to expand these spherical oscillator states $\psi_{n \ell \lambda}(\vec{r}, \hat{z})$ in terms of a cylindrically symmetric oscillator basis set, $\psi_{n_{z} n_{s} \lambda}(\vec{r}, \hat{z})$, using

$$
\psi_{n \ell \lambda}(\vec{r}, \hat{z})=\sum_{n_{z}} C_{n_{z} n_{s} \lambda}^{n \ell} \psi_{n_{z} n_{s} \lambda}(\vec{r}, \hat{z}) .
$$

Here the sum runs over all $n_{z}$ allowed by the constraint $2 n+\ell=n_{z}+2 n_{s}+\lambda$ imposed by the number of available oscillator quanta. This cylindrical representation allows an analytical evaluation of certain beam-directional integrals. These cylindrical oscillator wave function, with $\vec{r} \equiv(\vec{s}, z) \equiv$ $(s, \varphi, z)$, is given by

$$
\psi_{n_{z} n_{s} \lambda}(\vec{r}, \hat{z})=g_{n_{z} n_{s} \lambda} e^{-\alpha^{2}\left(z^{2}+s^{2}\right) / 2} H_{n_{z}}(\alpha z) h_{n_{s} \lambda}(\alpha \vec{s}) .
$$

Here

$$
h_{n_{s} \lambda}(\alpha \vec{s})=\frac{(-1)^{n_{s}} e^{i \lambda \varphi}}{\left(|\lambda|+n_{s}\right) !}(\alpha s)^{|\lambda|} L_{n_{s}}^{|\lambda|}\left(\alpha^{2} s^{2}\right),
$$

$H_{n_{z}}(\alpha z)$ is the Hermite polynomial, given by

$$
H_{n_{z}}(\alpha z)=n_{z} ! \sum_{q=0}^{\left\lfloor n_{z} / 2\right\rfloor}(-1)^{q} \frac{1}{q !\left(n_{z}-2 q\right) !}(2 \alpha z)^{n_{z}-2 q},
$$

the wave-function normalization constant $g_{n_{z} n_{s} \lambda}$ is

$$
g_{n_{z} n_{s} \lambda}=(-1)^{n_{s}} \alpha^{3 / 2}\left[\frac{n_{s} !\left(n_{s}+|\lambda|\right) !}{2^{n_{z}} n_{z} ! \pi^{\frac{3}{2}}}\right]^{1 / 2},
$$

and

$$
\alpha=\sqrt{\frac{M \omega_{0}(\delta)}{\hbar}} .
$$


The coefficients $C_{n_{z} n_{s} \lambda}^{n \ell}$ in the cylindrical expansion were obtained using the generating functions of the harmonic oscillator [39]. They are shown to be

$$
\begin{aligned}
C_{n_{z} n_{s} \lambda}^{n \ell}= & \frac{g_{n_{z} n_{s} \lambda}}{c_{n \ell}} n ! n_{z} ! \sqrt{\frac{2 \ell+1}{4 \pi}(\ell+\lambda) !(\ell-\lambda) !} \\
& \times\left[\sum_{p} \frac{(-1)^{p}}{2^{2 p-\lambda} p !(p-\lambda) !(\ell+\lambda-2 p) !}\right. \\
& \left.\times \frac{1}{\left(n_{s}+\lambda-p\right) !\left(n+p-n_{s}-\lambda\right) !}\right]
\end{aligned}
$$

and satisfy the symmetry relation

$$
C_{n_{z} n_{s}-\lambda}^{n \ell}=(-1)^{\lambda} C_{n_{z} n_{s} \lambda}^{n \ell}
$$

where

$$
c_{n \ell}=(-1)^{n} \alpha^{3 / 2} \sqrt{\frac{n !(2 \ell+2 n+1) ! !}{\pi^{5 / 2} 2^{\ell+n+2}}} .
$$

Our use of oscillator wave functions for the removed nucleons, which do not have the correct (Hankel function, for neutrons) asymptotic form, is expected to lead to slightly smaller nucleon removal cross sections than if using, for example, a bound eigenstate of a Woods-Saxon potential. The model is, however, reasonably accurate and applicable to cases where the removed nucleon is initially well bound, in which cases the reaction is sensitive to the wave function near its surface maximum and not to the asymptotic form of the bound state. Our (unavoidable) use of oscillator asymptotics is not expected to significantly affect the systematics of the sensitivities of reaction yields or the resulting residue momentum distributions to deformation. We comment on this sensitivity in Sec. IV B.

\section{B. Cross sections}

Single-nucleon removal reactions have been extensively used to both populate the states of and study the structure of light exotic nuclei with $A<40$. Most often these reactions have been treated using reaction models that assume (a) the valence nucleons are removed suddenly in fast interactions with the target, such that the reaction residue acts as a spectator and proceeds undisturbed, and (b) eikonal reaction dynamics, where the projectile is assumed to follow a straight-line path as it passes the target. In the vast majority of cases, the projectile has been assumed to be spherical.

The eikonal model expression for the stripping (inelastic breakup) cross section-that part of the projectile-target absorption cross section in which $A$ nucleons are transmitted and a nucleon excites the target-is expressed in terms of the projectile ground-state wave function, $\Phi_{I M}$, by, for example, Eq. (2) of Ref. [40]

$$
\sigma_{\mathrm{str}}=\frac{1}{2 I+1} \int d \vec{b} \sum_{M}\left\langle\left.\Phi_{I M}|| \mathcal{S}_{A}\left(\left\{\vec{b}_{A}\right\}\right)\right|^{2} A\left(b_{v}\right) \mid \Phi_{I M}\right\rangle
$$

Here $\mathcal{S}_{A}$ represents the $S$ matrix for the interactions of $A$ nucleons with the target, involving a set of impact parameters
$\left\{\vec{b}_{A}\right\}$, and an integral is carried out over all target-projectile center-of-mass impact parameters $\vec{b}$. The factor $A\left(b_{v}\right)$,

$$
A\left(b_{v}\right)=\left[1-\left|\mathcal{S}_{v}\left(b_{v}\right)\right|^{2}\right]
$$

is the (valence) nucleon absorption probability on the spherical target. Having made the spectator-core approximation, the stripping cross section for nucleon removal from a spherical state, $n \ell j$, the single-particle stripping cross section, is then $[10,40]$

$$
\sigma_{\mathrm{str}}^{n \ell j}=\frac{1}{2 j+1} \int d \vec{b}_{c}\left|\mathcal{S}_{c}\left(b_{c}\right)\right|^{2} \sum_{m}\left\langle\Psi_{n \ell j m}\left|A\left(b_{v}\right)\right| \Psi_{n \ell j m}\right\rangle,
$$

where the impact parameter integration variable has been changed to $\vec{b}_{c} . \mathcal{S}_{c}\left(b_{c}\right)$ is now the $S$ matrix for the core nucleus and $\langle\cdots\rangle$ indicates integration over the nucleoncore relative coordinate $\vec{r}$ and nucleon spin coordinates. In writing Eqs. (21) and (23) the (fast) adiabatic approximation was assumed, that the nucleon's position vector $\vec{r}$ in the projectile rest frame is assumed to be frozen for the short duration of the surface-grazing collisions with the target. The absolute value of the stripping single-particle cross section depends primarily on the radial extent of the single-nucleon wave function relative to the projectile core, as is largely determined by (a) the nucleon separation energy, (b) its orbital angular momentum $\ell$, and (c) the number of nodes in the radial wave function, $n=(N-\ell) / 2$. Weaker binding leads to a more extended nucleon wave function driving larger nucleon removal cross sections. The reaction residue parallel momentum distributions, $d \sigma_{\text {str }} / d k_{c}$, where $k_{c}$ is the momentum of the residue in the beam direction, are then characteristic of the orbital angular momentum of the removed nucleon [41].

We generalize this discussion for nucleon removal from an axially symmetric deformed projectile. We use the strong coupling limit, in which the nucleon wave function is taken to be a Nilsson state $v m$ for which $\pm m$ is the good quantum number. We assume the incident projectile ground state has spin $0^{+}$and is thus an unweighted average of the deformed intrinsic system over all $\hat{\Omega}$. Application of the adiabatic approximation now assumes that both $\vec{r}$ and the deformation degree of freedom $\hat{\Omega}$ are frozen during the collision. This step now connects the deformation of the core-target $S$ matrix, $S\left(\vec{b}_{c}, \hat{\Omega}\right)$, with that entering the projectile ground state. So, the spherical single-particle state is replaced with the Nilsson eigenstate $\Psi_{v m}$ for a fixed orientation $\hat{\Omega}$, the cross section is calculated for each orientation, and then the results must be averaged over all orientations (see also Eq. (2) of Ref. [29]). The stripping cross section now reads

$$
\sigma_{\mathrm{str}}^{v m}=\frac{1}{4 \pi} \int d \hat{\Omega} \int d \vec{b}_{c}\left|\mathcal{S}_{c}\left(\vec{b}_{c}, \hat{\Omega}\right)\right|^{2}\left\langle\Psi_{v m}\left|A\left(b_{v}\right)\right| \Psi_{v m}\right\rangle .
$$

The factor $\left|\mathcal{S}_{c}\left(\vec{b}_{c}, \hat{\Omega}\right)\right|^{2} A\left(b_{v}\right)$ in Eq. (24) has a simple intuitive interpretation, as the joint probability for elastic core-target scattering and an inelastic valence nucleon-target interaction at fixed $\vec{r}, \vec{b}_{c}$, and $\hat{\Omega}$, which must then be weighted by the nucleon's position probability, summed over all impact 


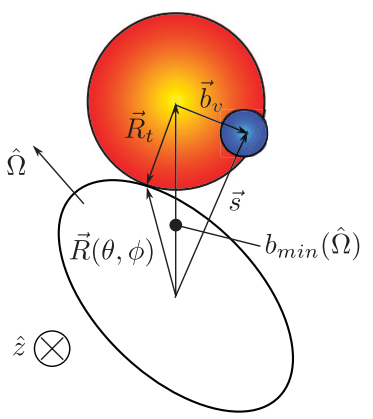

FIG. 4. (Color online) Impact parameter plane schematic showing the reaction coordinates. The allowed impact parameters for the spheroidal core is determined from that for all points on the surface $\vec{R}(\theta, \phi)$, where $\theta$ and $\phi$ are the polar angles of the surface point in the intrinsic system. The target radius is $R_{t}$. The maximum value of $b$ gives the minimum allowed impact parameter, $b_{\min }$ for a given projectile orientation. Note that the minimum allowable impact parameter does not necessarily occur where $\vec{R}_{t}$ is antiparallel to $R$.

parameters $\vec{b}_{c}$, and averaged over all projectile/residue orientations $\hat{\Omega}$. The cross sections for states with $\pm m$ are, of course, equal.

We now make the absorbing-disk approximation for the projectile core-target elastic $S$ matrix $[11,12]$. That is, any overlap of the pair during the reaction is assumed to lead to violent inelastic collisions that remove the residue from the one-nucleon removal channel. The target transit then selects a volume, grazing the core surface, in which the single-nucleon wave function is sampled. This is illustrated in Fig. 4. The core $S$ matrix is written as a step function, having a minimum impact parameter $b_{\min }$ for transmission, as

$$
\left|\mathcal{S}_{c}\left(\vec{b}_{c}, \hat{\Omega}\right)\right|^{2} \equiv \begin{cases}0 & \text { if } \quad b_{c}<b_{\min }(\hat{\Omega}) \\ 1 & \text { if } \quad b_{c} \geqslant b_{\min }(\hat{\Omega}) .\end{cases}
$$

For the deformed nuclei of interest here, the minimum impact parameter $b_{\min }(\hat{\Omega})$ naturally depends on the degree of deformation $\delta$ and, critically, on the orientation $\hat{\Omega}$ relative to the laboratory $z$ axis.

The shape of the spheroidal core, like the deformed oscillator potential, is chosen such that its volume is conserved as the deformation changes. The surface coordinates $(x, y, z)$ of the spheroid are defined by

$$
d_{0}(\delta)^{2}\left(1+\frac{2}{3} \delta\right)\left(x^{2}+y^{2}\right)+d_{0}(\delta)^{2}\left(1-\frac{4}{3} \delta\right) z^{2}=R_{c}^{2},
$$

where $d_{0}(\delta)$ has the same deformation dependence as $\omega_{0}(\delta)$, namely

$$
d_{0}(\delta)=\left(1-\frac{4}{3} \delta^{2}-\frac{16}{27} \delta^{3}\right)^{-1 / 6} .
$$

Though the volume of the solid spheroid is independent of deformation, its root-mean-squared (rms) radius, $\left\langle r^{2}\right\rangle_{c}^{\frac{1}{2}}$, is not and, to second order, the mean-squared radius of the spheroidal core is

$$
\left\langle r^{2}\right\rangle_{c, \delta}=\left\langle r^{2}\right\rangle_{c, \delta=0}\left(1+\frac{4}{9} \delta^{2}+\frac{32}{81} \delta^{3}+\cdots\right) .
$$

Over the range of deformations considered here (up to $\delta=0.4),\left\langle r^{2}\right\rangle_{c, \delta}^{\frac{1}{2}}$ of the core increases by $\approx 5 \%$.
For the nucleon-target $S$ matrix, where the diffuse surface of the target is important for determining the magnitude of the nucleon removal cross section, we use the single-folding model, a finite-ranged effective nucleon-nucleon interaction, and the target density. The range of impact parameters $b_{v}$ over which the nucleon $S$-matrix transitions from being small to of order unity, which determine $A\left(b_{v}\right)$, is larger for the valence nucleon than for the core, as fractions of the most important surface-grazing impact parameters. The calculated stripping cross sections are poorly described if one assumes a sharp-cutoff, absorptive-disk description of the nucleon-target interaction. The interaction of the valence nucleon and spherical target depends only on their relative positions and not the projectile orientation. In the core-target system we use a target size $R_{t}$, consistent with the folding model $S$ matrix for the nucleon-target interaction. We assume the target has a point nucleon density of Gaussian form. The finite-range nucleon-nucleon effective interaction is also assumed to have a Gaussian form-factor; thus, the evaluation of the nucleon-target $S$ matrix is, in fact, analytic and numerical folding is not required.

Making explicit the $z$ and transverse coordinate integrals over the nucleon's coordinate and applying the absorptive-disk approximation for the core-target $S$ matrix, the removal cross section from Nilsson model state $v m$ is therefore

$$
\begin{aligned}
\sigma_{\mathrm{bd}}^{v m}= & \frac{1}{4 \pi} \int d \hat{\Omega} \int_{b_{\min }(\hat{\Omega})}^{\infty} d \vec{b}_{c} \int d \vec{s} A\left(b_{v}\right) \\
& \times \int d z\left\langle\left|\Psi_{v m}(\vec{r}, \hat{\Omega})\right|^{2}\right\rangle_{\mathrm{sp}},
\end{aligned}
$$

where $\langle\cdots\rangle_{\text {sp }}$ now indicates integration over the spin coordinate of the nucleon. The distribution of the cross section with respect to the beam-directional residue momentum $k_{c}$ is found by taking the $z$-directional Fourier transform of the nucleon wave function; that is,

$$
\begin{aligned}
\frac{d \sigma_{\mathrm{bd}}^{v m}}{d k_{c}}= & \frac{1}{4 \pi} \int d \hat{\Omega} \int_{b_{\min }(\hat{\Omega})}^{\infty} d \vec{b}_{c} \int d \vec{s} A\left(b_{v}\right) \\
& \times\left\langle\left|\frac{1}{\sqrt{2 \pi}} \int d z e^{i k_{c} z} \Psi_{v m}(\vec{r}, \hat{\Omega})\right|^{2}\right\rangle_{\mathrm{sp}} .
\end{aligned}
$$

Upon inserting the wave function [Eq. (9)] into this expression, we obtain

$$
\begin{aligned}
\frac{d \sigma_{\mathrm{bd}}^{\nu m}}{d k_{c}}= & \frac{1}{4 \pi} \int d \hat{\Omega} \int_{b_{\min }(\hat{\Omega})}^{\infty} d \vec{b}_{c} \int d \vec{s} A\left(b_{v}\right) \\
& \times\left\langle\left|\Phi_{\nu m}\left(\vec{s}, k_{c}, \hat{\Omega}\right)\right|^{2}\right\rangle_{\mathrm{sp}},
\end{aligned}
$$

where the $z$-directional Fourier transform has been evaluated analytically, to give

$$
\begin{aligned}
\Phi_{v m}\left(\vec{s}, k_{c}, \hat{\Omega}\right) \equiv & \frac{1}{\sqrt{2 \pi}} \int d z e^{i k_{c} z} \Psi_{v m}(\vec{r}, \hat{\Omega}) \\
= & \sum_{n \ell j} a_{v m}^{n \ell j} \sum_{m^{\prime}} \mathcal{D}_{m^{\prime} m}^{j}(\hat{\Omega}) \sum_{\lambda \sigma}\left(\ell \lambda s \sigma \mid j m^{\prime}\right) \chi_{s \sigma} \\
& \times \sum_{n_{z} n_{s}} C_{n_{z} n_{s} \lambda}^{n \ell} g_{n_{z} n_{s} \lambda} \exp \left(-\alpha^{2} s^{2} / 2\right) h_{n_{s} \lambda}(\alpha \vec{s}) \\
& \times H_{n_{z}}\left(k_{c} / \alpha\right) i^{n_{z}} \exp \left(-k_{c}^{2} / 2 \alpha^{2}\right) / \alpha
\end{aligned}
$$


This form allows the momentum distributions to be calculated efficiently.

Our model is similar in some respects to the geometric model of Ref. [26], except that we use (a) a deformed coretarget $S$ matrix derived from an assumed quadrupole-deformed intrinsic shape, (b) a conventional density-based folding model for the nucleon-target $S$ matrix, and (c) Nilsson model calculations in an unstretched basis. In principle, the latter point should make little difference, because, once transformed from a stretched to an un-stretched basis, the Nilsson state wave functions will be identical save for minor differences arising from the added spin-orbit and angular momentum terms in the Nilsson Hamiltonian.

\section{Limit of small deformations}

We first discuss the zero-deformation limit of the model described above. Now the Nilsson potential is simply a spherical modified oscillator, the eigenstates of which are pure $(n \ell j m)$ spherical states. The core is spherical and within the absorptive-disk model the minimum impact parameter is independent of orientation and given simply by the sum of the core and target radii $b_{\min }=R_{t}+R_{c}$. In the calculation of the cross section, the only dependence on the orientation of the projectile symmetry axis is then in the rotation matrices of the rotated wave function in Eq. (9). The quantity of interest is the square modulus of the single-nucleon wave function averaged over the symmetry axis orientations $\hat{\Omega}$ that can now be evaluated analytically, using [42]

$$
\begin{aligned}
& \int d \alpha \int \sin \beta d \beta \mathcal{D}_{m^{\prime} m}^{j *}(\alpha, \beta, 0) \mathcal{D}_{n^{\prime} n}^{k}(\alpha, \beta, 0) \\
& =\frac{4 \pi}{2 j+1} \delta_{j k} \delta_{m n} \delta_{m^{\prime} n^{\prime}} .
\end{aligned}
$$

Here $(\alpha, \beta)$ are two of the Euler angles, the third of which is redundant when axial symmetry is assumed. In the limit of zero deformation each Nilsson state $v m$ has a single contributing $(n \ell j)$ and thus,

$$
\begin{aligned}
& \frac{1}{4 \pi} \int d \hat{\Omega}\left|\Psi_{v m}(\vec{r}, \hat{\Omega})\right|^{2} \\
& \quad=\frac{1}{4 \pi} \int d \hat{\Omega}\left|\sum_{m^{\prime}} \mathcal{D}_{m^{\prime} m}^{j}(\hat{\Omega}) \Psi_{n \ell j m^{\prime}}(\vec{r}, \hat{z})\right|^{2}
\end{aligned}
$$

Expanding the square modulus and averaging over orientations then gives

$$
\frac{1}{4 \pi} \int d \hat{\Omega}\left|\Psi_{v m}(\vec{r}, \hat{\Omega})\right|^{2}=\frac{1}{2 j+1} \sum_{m^{\prime}}\left|\Psi_{n \ell j m^{\prime}}(\vec{r}, \hat{z})\right|^{2} .
$$

The result, the average over the projections of a single spherical component $\left(n \ell j m^{\prime}\right)$, is identical to the conventional spherical model [see Eq. (23)]. For zero deformation our model gives the correct spherical limit. Similarly, averaging the single projection $m$ on the orientation axis over all orientations, we should obtain the spherical model cross sections and residue momentum distributions.

An intuitive expectation for small deformations will also prove useful. For small deformations it is reasonable to assume that the shape of the core, for $S$-matrix purposes, is approximately spherical, allowing us to perform the same average over orientation angles as above. It is also expected that the mixing of states in the Nilsson wave functions be confined to a single major shell. Carrying out the same averaging procedure as before, the $\delta_{j j^{\prime}}$ Kronecker $\delta$ that enters also implies $\delta_{n n^{\prime}}$ and $\delta_{\ell \ell^{\prime}}$, because within a single major shell each $j$ value has unique $n$ and $\ell$ attributes. Once again we obtain an average over the projections $m^{\prime}$, as for the spherical symmetry case,

$$
\frac{1}{4 \pi} \int d \hat{\Omega}\left|\Psi_{v m}(\vec{r}, \hat{\Omega})\right|^{2}=\frac{1}{2 j+1} \sum_{n \ell j m^{\prime}}\left[a_{v m}^{n \ell j}\right]^{2}\left|\Psi_{n \ell j m^{\prime}}(\vec{r}, \hat{z})\right|^{2},
$$

though now there are (incoherent) contributions from the contributing spherical components with weights given by the Nilsson wave-function amplitudes. One could, additionally, sum the above expression over all $N=2$ shell Nilsson states $v m$ in which case the normalization of the coefficients $\sum_{v m}\left[a_{v m}^{n \ell j}\right]^{2}$ then removes any dependence of the total removal cross section on the deformation $\delta$, save for small changes in the oscillator frequency owing to volume conservation. The total removal cross section is thus expected to be approximately constant for small deformations.

In this small deformations limit, changes to the cross sections and momentum distribution can be seen to be the result of the evolving spherical-basis structure of the Nilsson states. Consequently, wave functions that mix orbital angular momenta will exhibit the largest changes. In the $s d$ shell we expect therefore that changes to the $m=1 / 2$ Nilsson states will be largest. For small deformations the cross sections and momentum distributions for removal from $m=3 / 2$ and $m=5 / 2$ states, which are both purely $d$ wave, are expected to remain approximately constant and very similar to the spherical $d$-wave results. We emphasize that these simple expectations are relevant to cross sections that are inclusive to the full rotational band. Individual final states might still exhibit characteristic momentum distributions, but such features are beyond the calculations of the present paper.

\section{APPLICATION TO THE $s d$ SHELL: $A=28$ SYSTEMS}

We discuss in some detail the results obtained using the absorptive-disk model described above. In particular, we consider the sensitivity of cross sections and momentum distributions to (a) varying the projectile deformation, (b) including major shell mixing in the Nilsson model calculations, and (c) the orientation dependence of the core-target $S$ matrix.

Deformation has long been known to be prevalent in the middle of the $s d$ shell, and was more recently identified in exotic $s d-p f$ shell isotopes near the island of inversion. Given the limitations of the oscillator basis as regards the single-particle wave-function asymptotics and the absence of explicit separation energies, we consider generic calculations applicable to nuclei in this region, nominally for the $A=28$ 
systems. The even-even $A=28$ isobars have deformations estimated from their ground-state to first $2^{+}$-state $B(E 2)$ values; for example, ${ }^{28} \mathrm{Ne} \beta=0.36 \pm 0.03$ [43], ${ }^{28} \mathrm{Mg} \beta=$ $0.491 \pm 0.035$ [19], and ${ }^{28} \mathrm{Si} \beta=0.407 \pm 0.007$ [19]. To first order, these $\beta$ correspond to $\delta=0.34,0.46$, and 0.39 , respectively. The measured quadrupole moments of ${ }^{28} \mathrm{Al}$ and ${ }^{28} \mathrm{Na}$ are also indicative of sizable deformation [44].

For completeness we calculate removal cross sections for all $N=2$ Nilsson states, that is, the [220]1/2, [211]3/2, [202]5/2, [211]1/2, [200]1/2, and [202]3/2 configurations, though not all will be active in any given physical example. We reiterate that our results, following integration over orientations, are inclusive with respect to the final states of the residual nuclei for the Nilsson state in question. Comparisons with experiment should thus be restricted to such data.

\section{A. Calculation parameters}

The input parameters to the model are as follows. The Nilsson model Hamiltonian uses a spin-orbit parameter $\kappa=$ 0.05 and for the centrifugal term the values $\mu=0$ for $N \leqslant 2$ and $\mu=0.45$ for $N>2$. These are similar to the values used by Ref. [35]. The sensitivity to these parameters is touched upon in Sec. V. Our calculations use major oscillator shells up to $N=8$, and we present results for both a pure $s d$-shell $(N=2)$ model space and when mixing the $N=0-4$ and $N=0-8$ major shells. The radial wave functions use the oscillator frequency parametrization of Ref. [45], giving $\hbar \omega_{0}(\delta=0)=12.108 \mathrm{MeV}$ for $A=28$, and a corresponding $\left\langle r^{2}\right\rangle^{\frac{1}{2}}=3.46 \mathrm{fm}$ for $N=2$. Spherical Hartree-Fock calculations using the Skyrme SkX interaction [46] generate $0 d_{5 / 2}$, $0 d_{3 / 2}$, and $1 s_{1 / 2}$ orbital rms radii of $3.330,3.546$, and $3.454 \mathrm{fm}$, respectively, in good agreement with our oscillator value. Volume conservation of the deformed oscillator makes $\hbar \omega_{0}$ weakly deformation-dependent and consequently there is a systematic decrease in the $\left\langle r^{2}\right\rangle^{\frac{1}{2}}$ of the Nilsson state as deformation increases, superimposed on the changes that arise from mixing between different major shells.

For the nucleon-target interaction we have used a singlefolding of the nucleon with the point-nucleon density of the ${ }^{9}$ Be target. This density was assumed to be of Gaussian form with an rms radius of $2.36 \mathrm{fm}$. A Gaussian finite-ranged effective nucleon-nucleon interaction with range $0.5 \mathrm{fm}$ was assumed in the folding with strength determined by the free nucleon-nucleon cross sections, taken from the parametrization of Ref. [47]. The resulting nucleon-target reaction cross section at $65 \mathrm{MeV}$ per nucleon is $284 \mathrm{mb}$, in reasonable agreement with available experimental values (for the proton${ }^{9} \mathrm{Be}$ system [48]). We note that this folded nucleon-target interaction and its derived $S$ matrix are the only explicitly projectile energy-dependent inputs to the calculations that follow. As the nucleon-target reaction cross section falls only slowly (and smoothly) with the incident energy the results we present, at $65 \mathrm{MeV}$ per nucleon, will be rather energy-independent, other than a gradual overall reduction common to all of the calculated nucleon removal cross sections.
For the core-target interaction, we have chosen core $R_{c}$ and target $R_{t}$ radii to reproduce the estimated nucleontarget and core-target reaction cross sections, appropriate for $65 \mathrm{MeV} /$ nucleon. The effective target radius $R_{t}$ is chosen to be $3.00 \mathrm{fm}$, giving a neutron- ${ }^{9}$ Be reaction cross section of $283 \mathrm{mb}$, consistent with that of the folding-model interaction used for the eikonal nucleon-target $S$ matrix. To determine the core radius $R_{c}$ we first used the empirical nucleus-nucleus reaction cross section parametrization of Kox et al. [49] to estimate a $\sigma_{R}=1344 \mathrm{mb}$ for the ${ }^{27} \mathrm{Al}-{ }^{9} \mathrm{Be}$ system. From this we deduce a minimum (spherical) impact parameter $b_{\text {min }}=R_{t}+R_{c}=6.54 \mathrm{fm}$ and thus a spherical core radius $R_{c}=3.54 \mathrm{fm}$. The root-mean-squared radius of a sharp-cutoff core is then $\left\langle r^{2}\right\rangle_{c}^{\frac{1}{2}}=\sqrt{3 / 5} R_{c}=2.74 \mathrm{fm}$, whereas spherical Hartree-Fock calculations using the $\mathrm{SkX}$ interaction [46] give $\left\langle r^{2}\right\rangle_{c}^{\frac{1}{2}}=2.95 \mathrm{fm}$ for ${ }^{27} \mathrm{Al}$. However, if we use this HartreeFock $A=27$ density distribution and a double-folding model calculation for the ${ }^{27} \mathrm{Al}-{ }^{9} \mathrm{Be}$ interaction, then we calculate a reaction cross section of $1387 \mathrm{mb}$, within $4 \%$ of the empirical estimate of the the sharp-cutoff approximation used here. We conclude that the values $R_{c}=3.54 \mathrm{fm}$ and $R_{t}=3.0$ fm provide parameters for the ${ }^{27} \mathrm{Al}-{ }^{9} \mathrm{Be}$ system that describe realistically the core and target sizes and their reaction cross sections and that are also consistent with the neutron- ${ }^{9}$ Be $S$ matrix used.

\section{B. Spherical calculations}

To assess the accuracy of the black-disk approximation, we first make comparative spherical model calculations for our mass 28 example, ${ }^{28} \mathrm{X}(-1 \mathrm{n})$, using the folding-model approach for the $S$ matrices, using the methodology and Hartree-Fock densities described above. We use the same harmonic oscillator wave functions as used for the deformed model results, with $\hbar \omega=12.108 \mathrm{MeV}$ [45]. With this prescription we obtain $1 s$ - and $0 d$-state stripping cross sections of 14.43 and $12.25 \mathrm{mb}$, respectively. We will not calculate the diffraction cross section-nucleon removal owing to elastic breakup - in the absorptive-disk calculations, but these spherical folding-model calculations give $\sigma_{\text {diff }}=4.36$ and $3.26 \mathrm{mb}$ for the $1 s$ and $0 d$ states, respectively.

Assuming the sharp, absorptive-disk approximation to the (spherical) core-target $S$ matrix the resulting stripping cross sections are smaller, giving $1 s$ - and $0 d$-state $\sigma_{\text {str }}=11.81$ and $9.38 \mathrm{mb}$, reductions of $18 \%$ and $23 \%$ on the folding-model approach. This feature of absorptive-disk cross sections was known [10], so our calculations will systematically underestimate cross sections by $\approx 20-30 \%$. This does not impact our discussions of the qualitative trends in cross sections and parallel momentum distributions with changes in deformation for which the absorptive-disk model is applicable and contains the essential physical inputs.

The oscillator wave functions have incorrect asymptotics. For comparison we calculate $s$ - and $d$-wave radial wave functions using a Woods Saxon potential. The radius and depth parameters are fit to reproduce the ${ }^{28} \mathrm{Al}$ neutron separation energy, $S_{n}=7.725 \mathrm{MeV}$, and the same $\left\langle r^{2}\right\rangle^{\frac{1}{2}}$ of the oscillator 


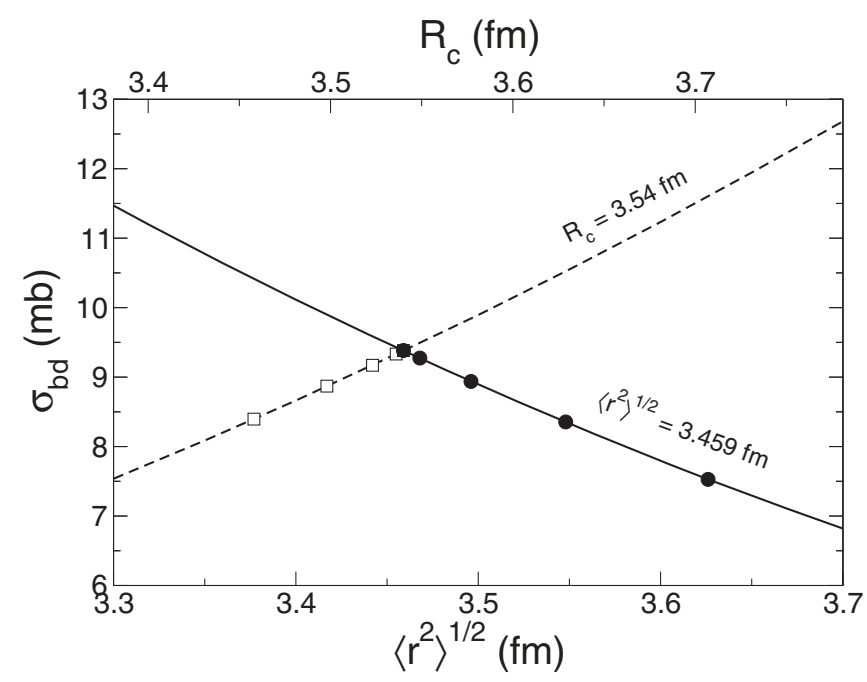

FIG. 5. Neutron removal cross sections calculated (i) for $0 d$-state oscillator wave function with different $\left\langle r^{2}\right\rangle^{\frac{1}{2}}$ (dashed) assuming a fixed spherical core with $R_{c}=3.54 \mathrm{fm}$ and (ii) for different $R_{c}$ and a fixed orbital $\left\langle r^{2}\right\rangle^{\frac{1}{2}}=3.46 \mathrm{fm}$ (solid). From right to left the squares indicate $\left\langle r^{2}\right\rangle^{\frac{1}{2}}$ sizes corresponding to $\delta=0,0.1,0.2,0.3$, and 0.4 , derived from Eq. (3). Similarly, but from left to right, the circles indicate the core sizes, derived from $R_{c}=\sqrt{5 / 3}\left\langle r^{2}\right\rangle_{c}^{\frac{1}{2}}$ and Eq. (28), for $\delta=0,0.1,0.2,0.3$, and 0.4 .

wave functions, with a diffuseness $a=0.6 \mathrm{fm}$. Again using the black-disk approximation, the resulting cross sections are 12.33 and 10.07 for $s$ and $d$, respectively, such that our use of oscillator wave functions underestimates the cross sections by a modest $5 \%-7 \%$.

Because the reaction samples the nucleon wave function near to and at the projectile surface, it is sensitive to both (a) the $\left\langle r^{2}\right\rangle^{\frac{1}{2}}$ of the nucleon's radial wave function and (b) the shape and $\left\langle r^{2}\right\rangle_{c}^{\frac{1}{2}}$ of the core. Because deformation will change both of these essential sizes, the former in a state-dependent manner, we first consider their sensitivities within the spherical limit. For a fixed core size, nucleon removal cross sections are known to essentially scale linearly with the rms radius of the nucleon wave function (see, e.g., Fig. 2 of Ref. [16]). We obtain the same result when using the absorptive-disk approximation described above, as is shown in Fig. 5. A similar (linear) $R_{c}$ sensitivity is found if one considers a fixed orbital $\left\langle r^{2}\right\rangle^{\frac{1}{2}}$ and target size $R_{t}$ but varies $R_{c}$; now the cross section falls essentially linearly with $R_{c}$. Thus, in a spherical model, the reduction in the rms radius of the $N=2$ neutron states over the range of deformations considered leads to an $\approx 10 \%$ reduction in the $0 d$-state removal cross section, from 9.38 to $8.40 \mathrm{mb}$.

The momentum distributions obtained using the absorptivedisk approximation (and $\delta=0$ ) are compared to those calculated [50] using the folding-model-derived $S$ matrices in Fig. 6. Excellent agreement is found for both the $1 s$ - and the $0 d$ state distributions. Thus, the absorptive-disk approximation to the core-target $S$ matrix is also applicable for study of

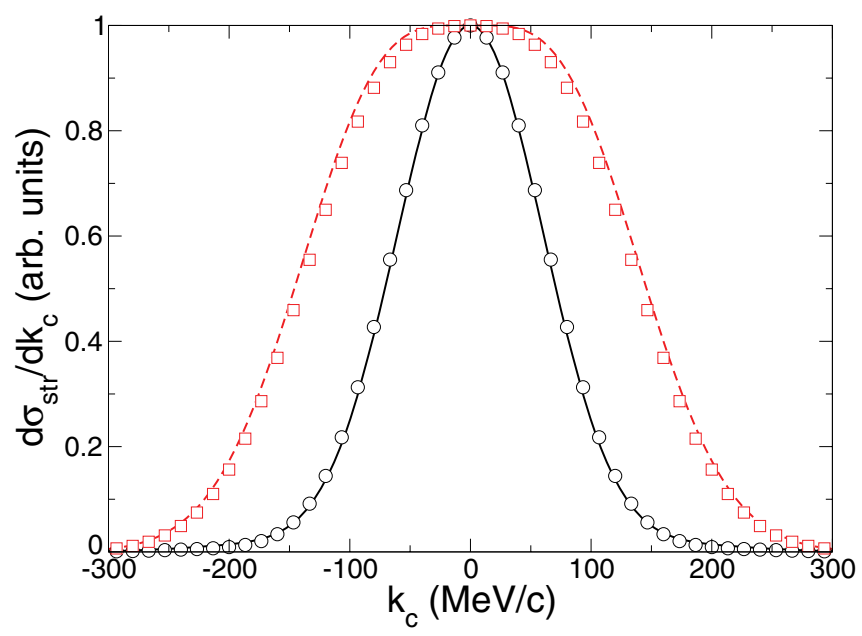

FIG. 6. (Color online) Calculations for $1 s$ - (black solid line, circles) and $0 d$-state (red dashed line, squares) nucleon removal using a folding model $S$-matrix for the projectile core (solid lines) and the absorptive-disk approximation (open symbols). The $1 s(0 d)$ absorptive-disk calculations are marginally wider (narrower) than those using the folding-model core-target $S$ matrix, but the overall agreement is excellent.

the systematic, deformation-induced changes to momentum distributions.

\section{Deformed model results}

We now discuss the effects arising from deformation with the model assumptions and parameters detailed above. There are several effects. Cross sections are given for (i) spherical and spheroidal core shapes and (ii) for Nilsson model spaces spanning $N=2, N=0-4$, and $N=0-8$. We discuss their effects on neutron removal from each $N=2$ Nilsson state according to their asymptotic quantum numbers. For each we show the absorptive-disk cross sections $\sigma_{\mathrm{bd}}$, and the properties of the state as a function of the assumed deformation $\delta$.

As mentioned above, previous (spherical) studies showed that the single-particle cross sections scale approximately linearly with the rms radius, $\left\langle r^{2}\right\rangle^{\frac{1}{2}}$, of the nucleon's wave function, larger radii extending the nucleon's position probability beyond the absorptive core radius and increasing its probability of interactions with the target. In the presence of deformation this simple picture becomes more complex. Though $\left\langle r^{2}\right\rangle^{\frac{1}{2}}$ of the bound states will change with deformation, so will the shapes of the absorptive spheroidal core and the nucleon wave functions relative to the core. Consequently, the larger $\left\langle r^{2}\right\rangle^{\frac{1}{2}}$, owing to an increased deformation, do not necessarily make the nucleon more accessible to collisions with the target.

To allow a qualitative interpretation of the calculated cross sections, we plot both $\left\langle r^{2}\right\rangle^{\frac{1}{2}}$ of the nucleon wave function and the average orbital angular momentum content of the state, $\langle\ell\rangle$, computed as

$$
\langle\ell\rangle_{v m}=\sum_{n \ell j}\left[a_{v m}^{n \ell j}\right]^{2} \cdot \ell .
$$




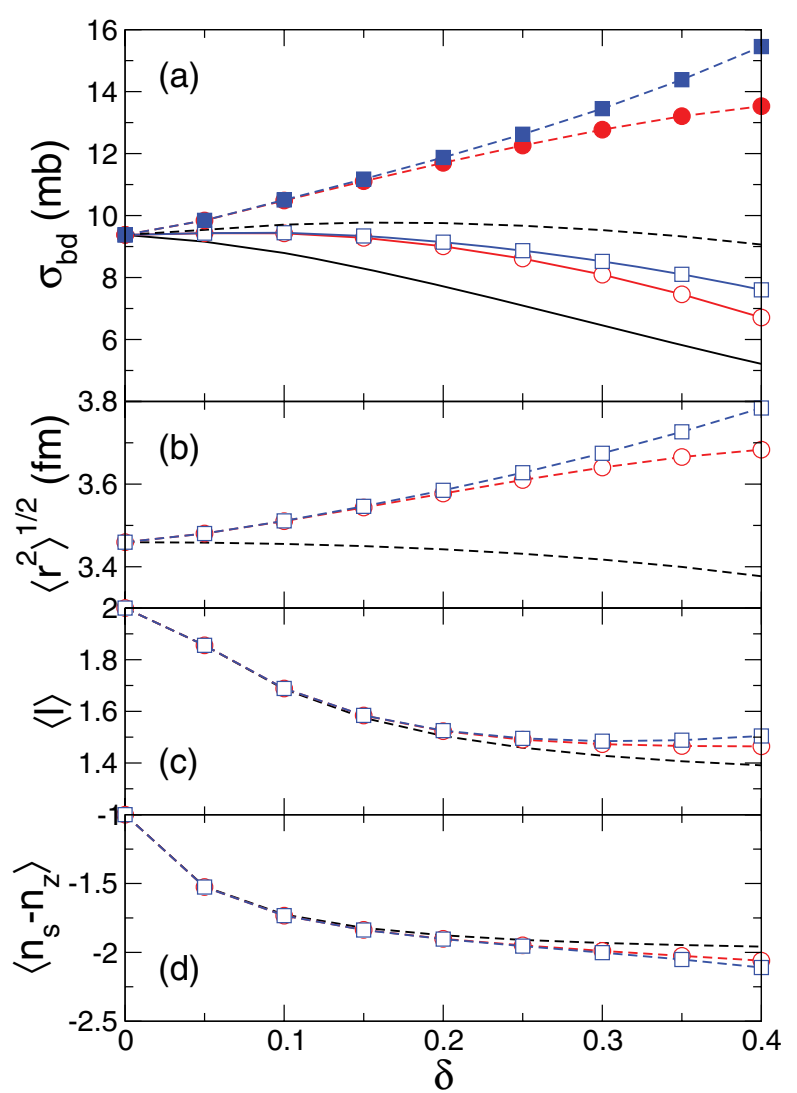

FIG. 7. (Color online) Calculations for the [220]1/2 Nilsson state. (a) Cross sections $\sigma_{\mathrm{bd}}$ as a function of deformation $\delta$, with a spherical (dashed lines) and a spheroidal core (solid lines). Results are for $N=2$ (black lines), $N=0-4$ (red lines, circles), and $N=0-8$ (blue lines, squares) Nilsson model spaces. (b) The wave-function rms radius, (c) the average $\ell$ value of the state, (d) $\left\langle n_{s}-n_{z}\right\rangle$, for the model spaces defined in (a).

For prolate deformations we also expect the cross sections for Nilsson states that are spatially extended along the symmetry axis to be smaller and those that extend in the perpendicular directions to be larger. It is therefore also useful to show the quantity

$$
\left\langle n_{s}-n_{z}\right\rangle_{v m}=\sum_{n_{z} n_{s} \lambda}\left[a_{v m}^{n_{z} n_{s} \lambda}\right]^{2} \cdot\left(n_{s}-n_{z}\right)
$$

that for pure $N=2$ Nilsson states take asymptotic values -2 , $-1,0$, and 1 . Negative values indicate prolate-like states, well aligned with the core shape, and the larger values indicate oblate-like states.

\section{1. $[220] 1 / 2$}

The cross sections $\sigma_{\mathrm{bd}}$ and wave-function properties of the [220]1/2 Nilsson state are shown in Fig. 7 as a function of deformation $\delta$. As previously discussed, the spherical core $\sigma_{\mathrm{bd}}$ scale with the rms radius of the wave function and are further enhanced by the moderate decreases in $\langle\ell\rangle$. The $\sigma_{\mathrm{bd}}$ sensitivity for the spheroidal core calculations is more complex. For small $\delta$, the $\sigma_{\text {bd }}$ for the different Nilsson model

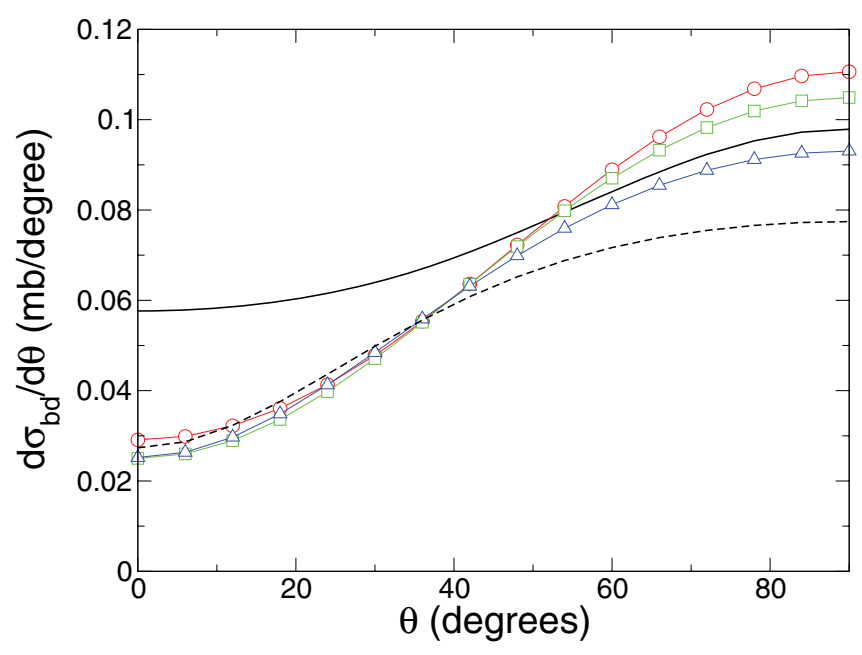

FIG. 8. (Color online) Cross section as a function of the angle $\theta_{\Omega}$ of the core symmetry axis, for the [220]1/2 Nilsson state. The lines show the results for deformations $\delta=0$ (solid black line), 0.1 (red line, circles), 0.2 (green line, squares), 0.3 (blue line, triangles), and 0.4 (dashed black line).

spaces are differentiated by their respective $\left\langle r^{2}\right\rangle^{\frac{1}{2}}$. At larger deformations cross sections for the prolate-type state, with $n_{z}=2$ asymptotically, decrease, as the valence nucleon wave function is masked by the core. The masking is more effective for the larger model spaces, the cross sections for which get progressively closer to that for the pure $N=2$ space.

This progressive decrease in the cross sections using the spheroidal core can be understood as an increased similarity of the core and nucleon wave-function shapes. Figure 8 shows the differential cross section $d \sigma_{\mathrm{bd}}(\delta) / d \theta_{\Omega}$ as a function of the angle $\theta_{\Omega}$ of the symmetry axis from the $z$ direction. At zero deformation, the cross section is small for small $\theta_{\Omega}$ and large for large $\theta_{\Omega}$, owing to the prolate $\left(\left\langle n_{s}-n_{z}\right\rangle=-1\right)$ nature of the $\delta=0$ state, which is a mixture of $\lambda=0$ and 1 components. The sudden changes in the cross sections for small $\delta$ reflect a rapidly changing shape of the nucleon wave function relative to the core [as shown by Fig. 7(d)]. The relative constancy of this quantity for $\delta>0.2$ leads to a reduction of the cross section for large $\theta_{\Omega}$ as increasing core deformation masks the nucleon further.

The momentum distributions for removal from the [220]1/2 state are shown in Fig. 9. The width of the distribution follows closely the changes in $\langle\ell\rangle$ [Fig. 7(c)], which drops from 2 at $\delta=0$ to $\approx 1.5$ at $\delta=0.2$, after which it is almost constant. This causes the width of the distribution to decrease rapidly on $\delta=0-0.2$, after which the width changes are small. A similar qualitative trend was also seen in the calculations of Ref. [26] (e.g., Fig. 9 of that reference) though the changes in shape are significantly smaller in our calculations.

\section{2. $[211] 3 / 2$}

The cross sections $\sigma_{\mathrm{bd}}$ and wave-function properties of the [211]3/2 state are shown in Fig. 10 as a function of $\delta$. The spherical core calculations now follow the trends of $\left\langle r^{2}\right\rangle^{\frac{1}{2}}$ 


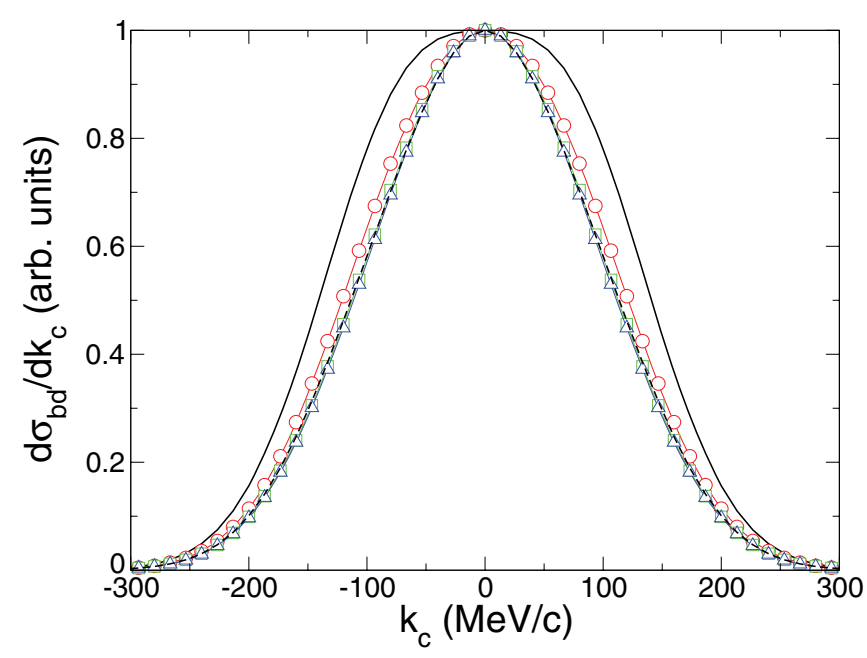

FIG. 9. (Color online) Residue momentum distributions following nucleon removal from the [220]1/2 state. The results correspond to deformations $\delta=0$ (black solid line), 0.1 (red solid line, circles), 0.2 (green solid line, squares), 0.3 (blue solid line, triangles), and 0.4 (black dashed line).

closely owing to the negligible changes in $\langle\ell\rangle$, the result of there being no $N=2$ in-shell mixing of orbital angular momenta. For the same reason the changes in $\left\langle n_{s}-n_{z}\right\rangle$ are also modest, but the prolate trend with increasing $\delta$ means the cross section gently falls with deformation for the reasons discussed above, despite the increasing $\left\langle r^{2}\right\rangle^{\frac{1}{2}}$ values. The momentum distributions for removal from the [211]3/2 state are shown in Fig. 11 for the spheroidal core and $N=0-8$ model space. This state is dominated by the $0 d, N=2$ configurations for all deformations, reflected in the $\delta$-independent momentum distribution shape. Very small changes in the [211]3/2 momentum distribution were also reported in Ref. [26], in qualitative agreement with the present results.

\section{3. $[202] 5 / 2$}

The [202]5/2 state has a pure $0 d_{5 / 2}$ configuration within the $s d$ shell. Changes to $\left\langle r^{2}\right\rangle^{\frac{1}{2}},\langle\ell\rangle$ and $\left\langle n_{s}-n_{z}\right\rangle$ are thus very small in comparison to the other states, resulting only from mixing between major shells. The [202]5/2 state cross sections and wave-function properties are shown in Fig. 12. Again the spherical core calculations track the modest $\left\langle r^{2}\right\rangle^{\frac{1}{2}}$ changes $(\approx 2 \%)$, with the spheroidal core cross sections increasing with $\delta$, because the state has $\left\langle n_{s}-n_{z}\right\rangle \approx 0$. This increase in the cross section, owing to the oblate nature of the state, can be understood with reference to $d \sigma_{\text {bd }}(\delta) / d \theta_{\Omega}$, shown in Fig. 13. In contrast to the prolate [220]1/2 state case of Fig. 8 , the cross section is now strongest for small $\theta_{\Omega}$, when the symmetry axis is aligned with the laboratory $z$ axis (the beam direction). As $\delta$ increases, more of the nucleon wave function is exposed to the target, enhancing the cross section at all orientations, but in particular for small $\theta_{\Omega}$. The purity of the $N=2$ configuration leads to an essentially constant

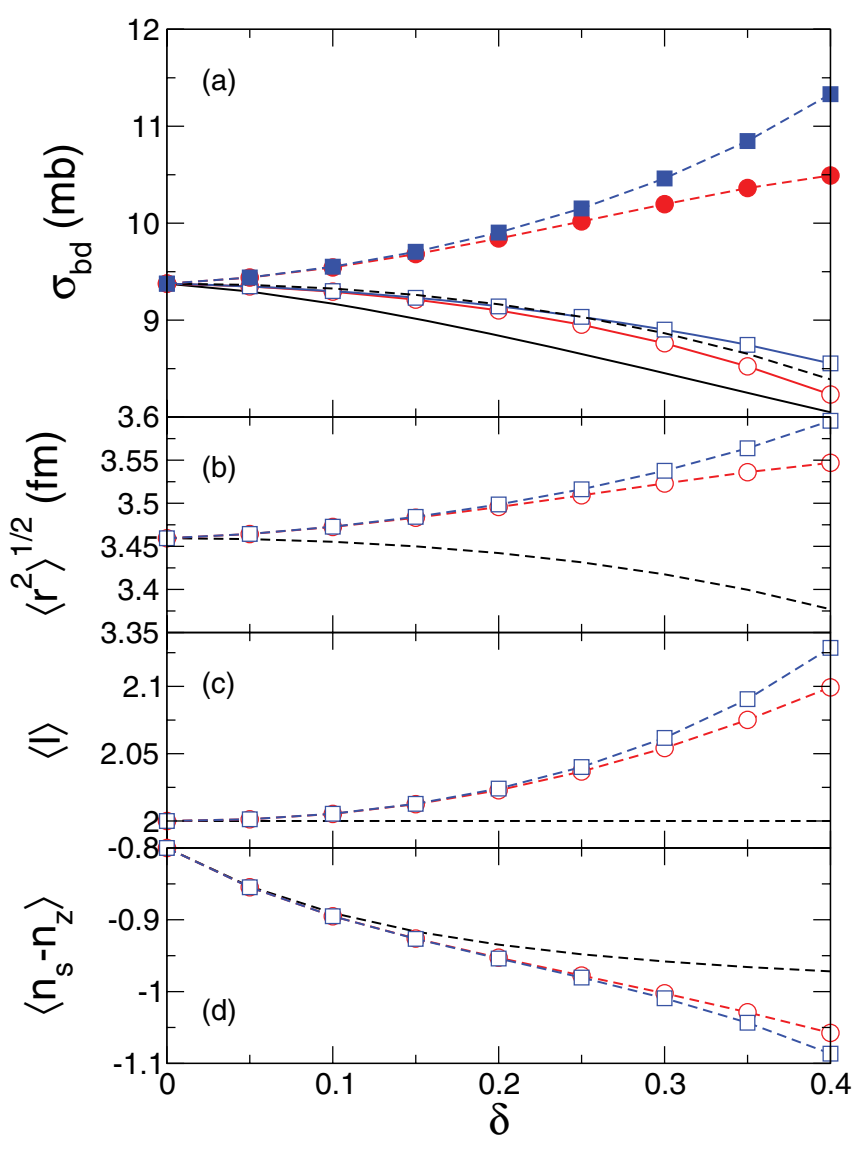

FIG. 10. (Color online) As for Fig. 7, but for the [211]3/2 Nilsson state.

$\langle\ell\rangle=2$ value and to a deformation-independent momentum distribution, essentially indistinguishable from that shown in Fig. 11.

In spherical nucleon removal models the residue momentum distributions depend strongly on the projections $m$

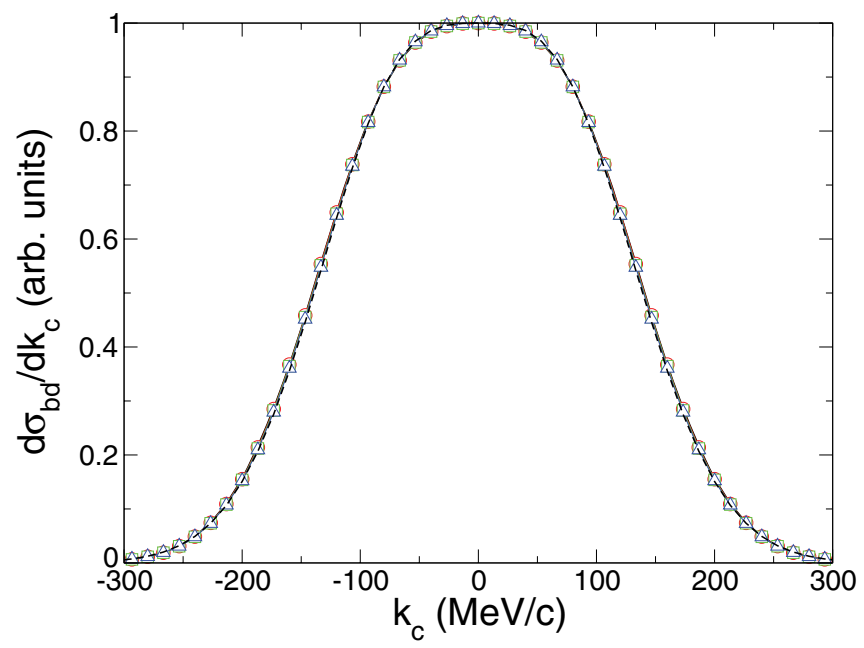

FIG. 11. (Color online) As for Fig. 9 but for the [211]3/2 Nilsson state. The momentum distributions are essentially independent of deformation. 


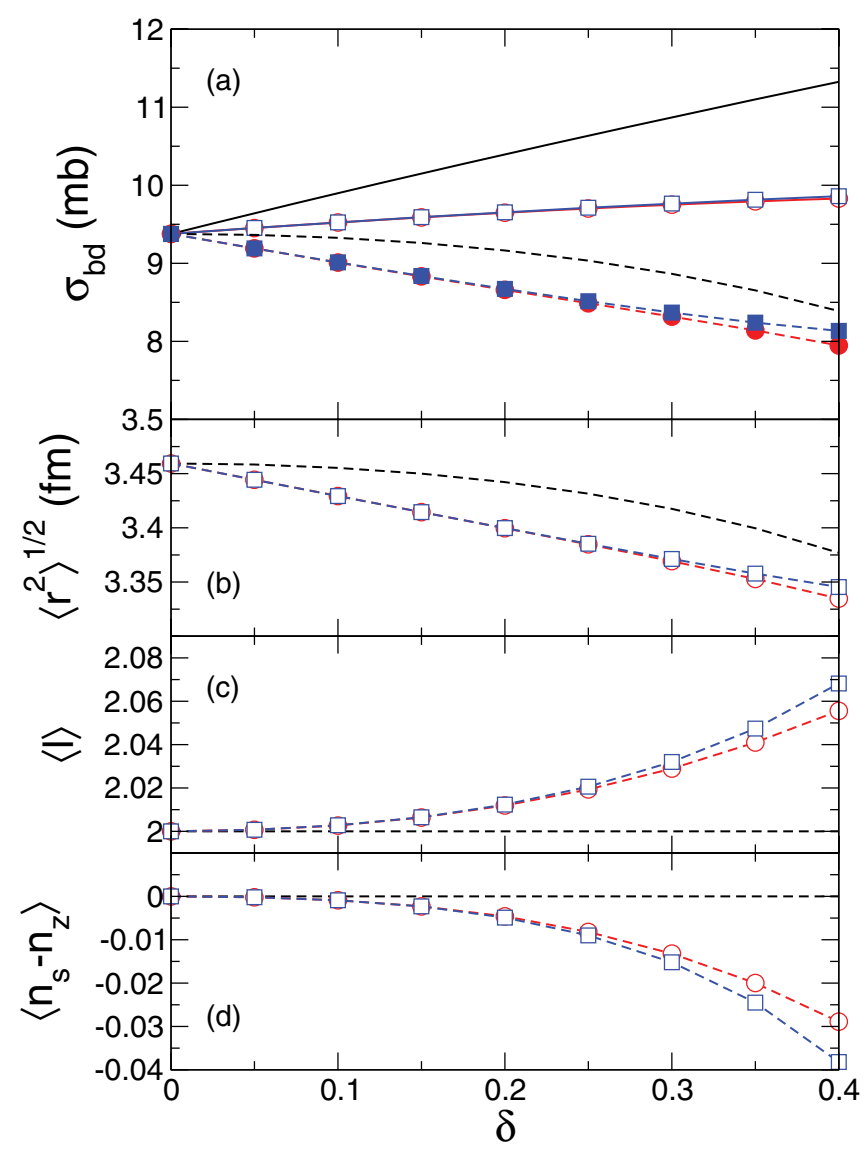

FIG. 12. (Color online) As Fig. 7 but for the [202]5/2 Nilsson state.

of the valence nucleon total angular momentum $j$ of the orbital [51]. Because the symmetry axis averaging process of the present model corresponds to an averaging over angular momentum projections in the spherical case, we would expect the calculated residue momentum distributions to depend strongly on $\theta_{\Omega}$. Figure 14 shows the calculated [202]5/2 state residue momentum distributions for fixed values of the symmetry axis $\theta_{\Omega}$ orientation, revealing this dependence. After averaging over orientations, the result is very similar to the pure spherical $0 d$-state calculation of Fig. 11. In principle, momentum distributions observed from a secondary beam of deformed nuclei could be altered if the secondary beam itself is produced with some degree of alignment, giving a nonisotropic $\hat{\Omega}$ distribution (see, e.g., Ref. [52]).

\section{4. $[211] 1 / 2$}

In Fig. 15 the [211]1/2 state $\sigma_{\mathrm{bd}}$ and wave-function properties are shown in as a function of $\delta$. This state, which originates in the spherical $1 s_{1 / 2}$ state, quickly transitions from pure $s$ state to strongly $d$ state as deformation increases, as asymptotically the state has $\lambda=1$. Consequently, the cross section falls very rapidly for small $\delta$, as $\langle\ell\rangle$ rapidly increases. The increasing $\left\langle r^{2}\right\rangle^{\frac{1}{2}}$ then enhances the cross sections of the spherical core calculations. With the spheroidal core the $\sigma_{\mathrm{bd}}$

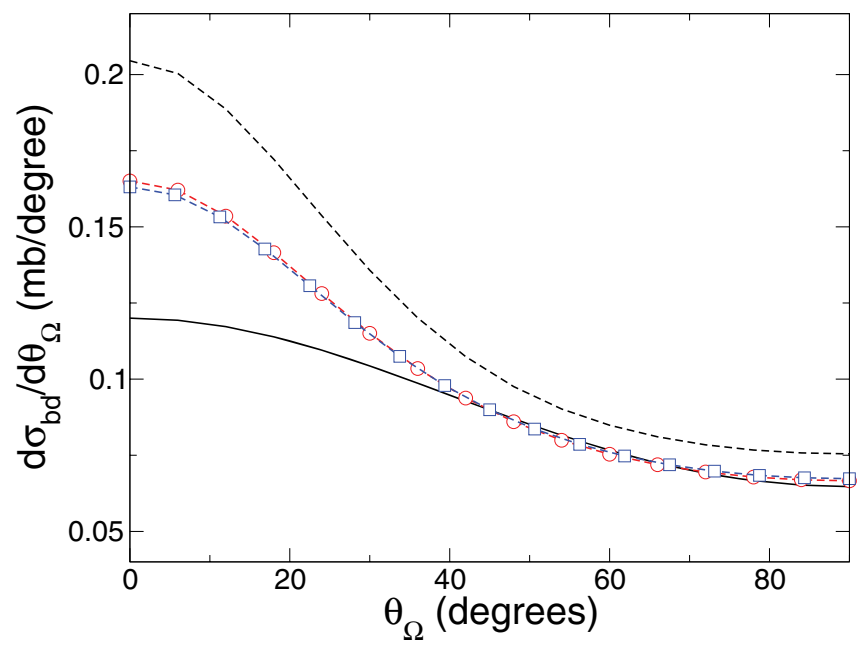

FIG. 13. (Color online) Cross section as a function of the angle $\theta_{\Omega}$ of the core symmetry axis for the [202]5/2 Nilsson state. The lines show the results for $\delta=0$ (solid black line) and 0.4 (dashed black line) for $N=2$, and $\delta=0.4$ for the $N=0-4$ (dashed red line, circles) and $N=0-8$ (dashed blue line, squares) model spaces. The state is dominated by the spherical $0 d_{5 / 2}$ component for all deformations. As deformation increases, the oblate nature of the state enhances cross sections for smaller angles $\theta_{\Omega}$, when the projectile symmetry axis is aligned with the beam direction. The larger model spaces act to moderate this trend, though the dependence on $\theta_{\Omega}$ is essentially unchanged.

continues to fall owing to the increasingly negative $\left\langle n_{s}-n_{z}\right\rangle$, in a very similar fashion to the $[211] 3 / 2$ state that shares the same asymptotic quantum numbers (cf. Fig. 10). The associated momentum distributions are shown in Fig. 16. Here there are dramatic changes in the widths of the distributions as $\delta$ increases. The $\langle\ell\rangle$ changes evolve the narrow distribution

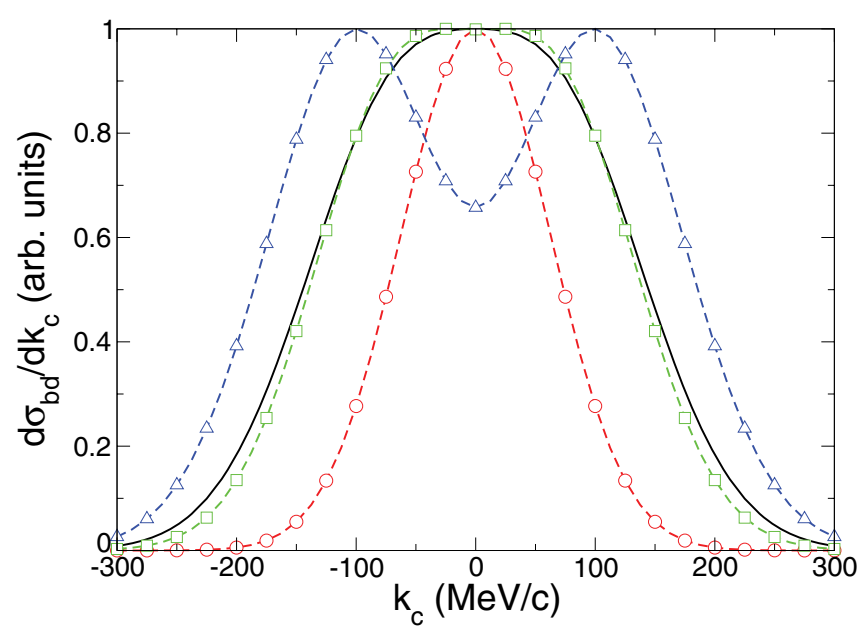

FIG. 14. (Color online) Momentum distributions for the [202]5/2 state with $\delta=0.4$, for fixed values of the symmetry axis $\theta_{\Omega}$. The $\theta_{\Omega}=$ $0^{\circ}$ (red dashed line, circles), $\theta_{\Omega}=45^{\circ}$ (green dashed line, squares), and $\theta_{\Omega}=90^{\circ}$ (blue dashed line, triangles) results are shown. The inclusive curve (black solid line) is the result when the correct angular average is performed. 


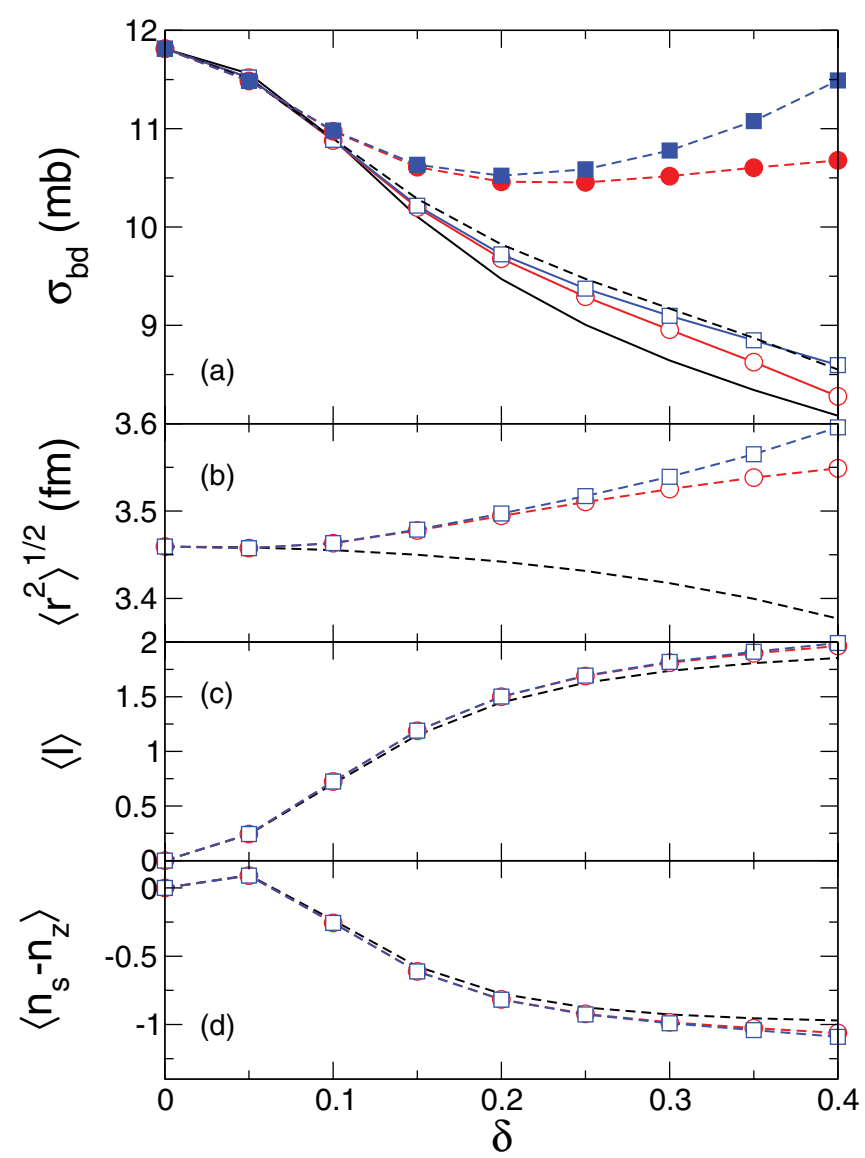

FIG. 15. (Color online) As for Fig. 7 but for the [211]1/2 Nilsson state.

at small deformation toward a broad distribution as the state evolves from an $s$-state to a $d$-state character. The qualitative trends agree with Ref. [26] (see Fig. 5 of that reference). The figure also shows the results obtained by weighting the spherical $1 s$ - and $0 d$-state momentum distribution calculations by the appropriate Nilsson model weights $\left[a_{v m}^{n \ell j}\right]^{2}$. The good agreement with the more sophisticated calculations, which incorporate the spheroidally deformed core $S$ matrix and a much larger Nilsson model space, indicates that that these momentum distribution shapes are largely the result of the contributions of the orbital angular momentum components present in the lowest order $(N=2)$ wave functions.

\section{5. $[200] 1 / 2$}

The results for the [200]1/2 asymptotic state are shown in Fig. 17. This state originates in the spherical $0 d_{3 / 2}$, rapidly mixes with the spherical $1 s_{1 / 2}$ state, enhancing the cross section at small deformations. The changes in $\langle\ell\rangle$ are then small for $\delta>0.2$, where the spherical core cross section then falls owing to the declining rms radius. This is the only $n_{s}=1$ state in the $N=2$ shell, and as a result the deformed core cross section increases markedly with deformation. The momentum distributions are shown in

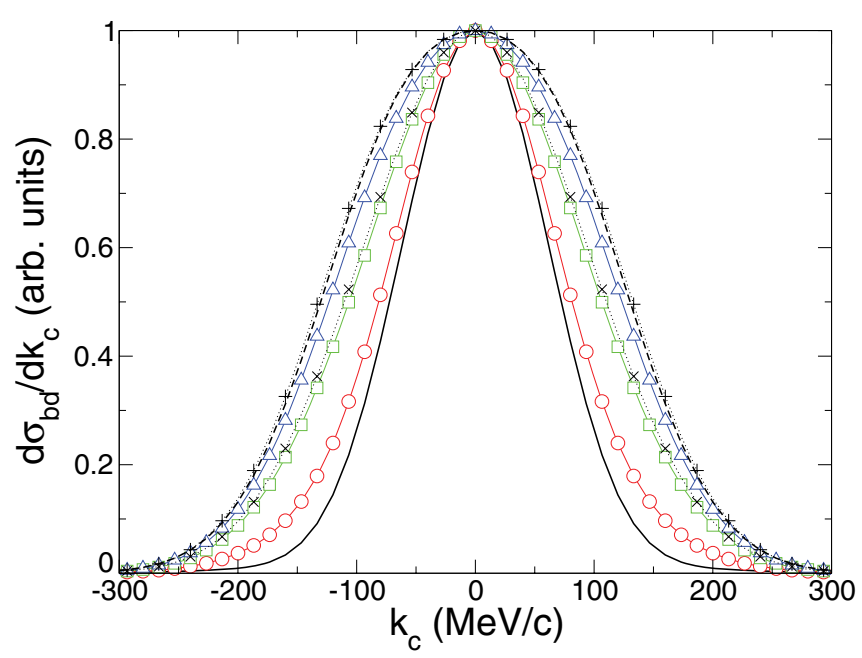

FIG. 16. (Color online) As for Fig. 9 but for the [211]1/2 Nilsson state. Additionally, the distributions obtained by adding the spherical $1 s$-state and $0 d$-state distributions, weighted by the $N=2$ model space Nilsson $\left[a_{v m}^{n \ell j}\right]^{2}$ coefficients appropriate for $\delta=0.2$ (dotted line, crosses) and $\delta=0.4$ (dotted line, plus signs) are shown. The agreement with the full calculations, the green squares and black dashed line, respectively, is good, indicating that the driver of the momentum distribution shapes is still the orbital angular momentum $\ell$, with relatively little impact from either couplings to higher oscillator shells or the use of a spheroidal core.

Fig. 18. The $\langle\ell\rangle$ for the state decreases from 2 to $\approx 0.8$ for the deformations shown and thus the momentum distribution decreases in width. The figure also compares the full $N=$ 0-8 model space results with the distributions obtained by weighting the $\ell=0$ and 2 spherical distributions with the Nilsson amplitudes $\left[a_{v m}^{n \ell j}\right]^{2}$ obtained from the pure $N=2$ model calculation. The agreement with the full calculation is reasonable, indicating that the effects of the altered wavefunction content and sampling induced by the larger model space and the spheroidal core, respectively, are relatively minor.

\section{6. $[202] 3 / 2$}

The results for the [202]3/2 asymptotic states are shown in Fig. 19. Similar to the $[211] 3 / 2$ and [202]5/2 state results, the weak mixing to other oscillator shells induces only small changes in $\langle\ell\rangle$ with $\delta$, such that the spherical core approximation tracks the changes in the rms radius. Indeed, because the the [202]3/2 and [202]5/2 states both originate in pure $0 d$ states and share the same asymptotic quantum numbers, the trends in $\sigma_{\mathrm{bd}}$ are very similar. With the spheroidal core, the small increase in $\left\langle n_{s}-n_{z}\right\rangle$ with $\delta$, and its asymptotic value of 0 , drive slowly increasing cross sections. For all deformations the state is dominated by $N=2$, $\ell=2$ components, such that this momentum distribution is insensitive to the deformation. The associated momentum distributions are essentially indistinguishable to those shown in Fig. 11 and hence are not shown. 


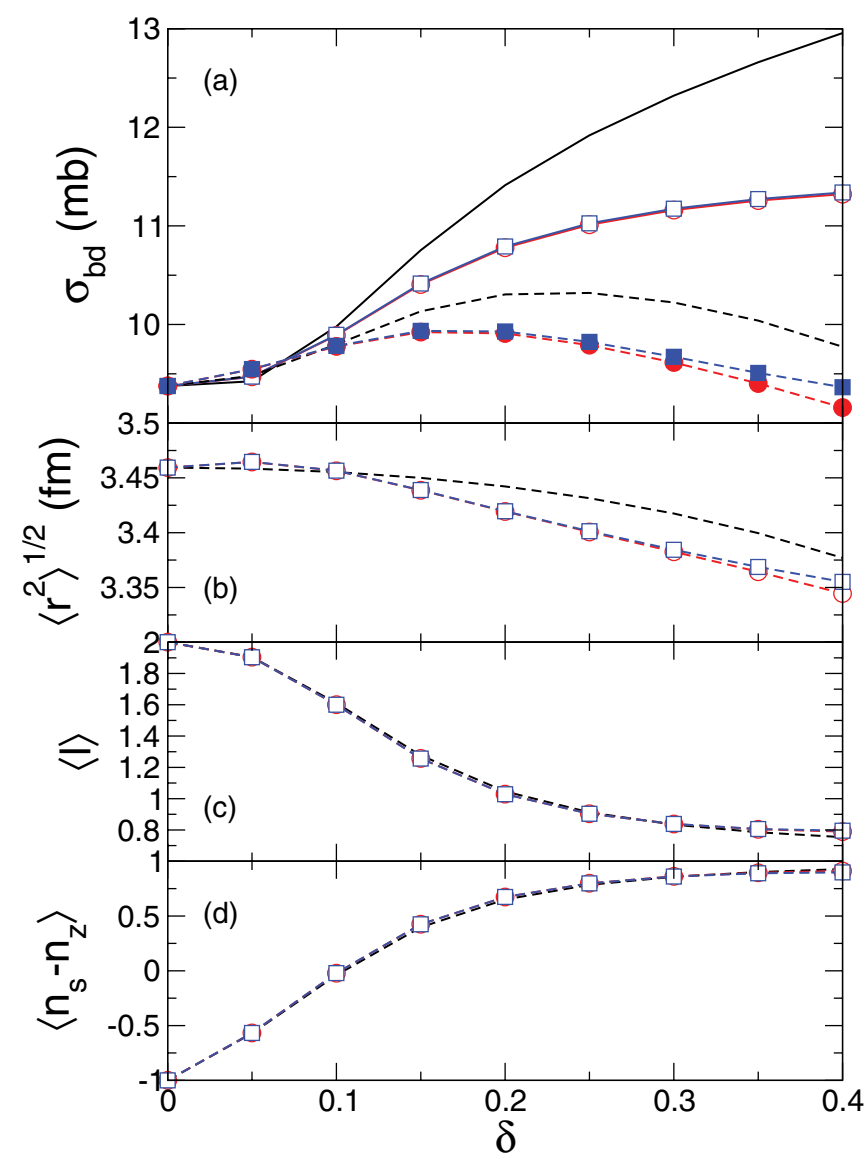

FIG. 17. (Color online) As for Fig. 7 but for the [200]1/2 Nilsson state.

\section{Summary}

To conclude this section we recap the results when using the $N=0-8$ model space and the spheroidal deformed core, collected in Fig. 20. Here the systematic trends are evident. (i) The changes in the cross section at small deformations largely reflect the changes in the orbital angular momentum composition of the Nilsson wave functions. Specifically, the cross sections have inverse trends to those of the $\langle\ell\rangle$, shown in panel (c). (ii) As deformation increases, the wave functions rapidly evolve toward their asymptotic forms, with relatively constant $\left\langle n_{s}-n_{z}\right\rangle$ for $\delta>0.2$. At this deformation, the cross section trends with increasing deformation are determined by $\left\langle n_{s}-n_{z}\right\rangle$, with larger values (oblate-like states) leading to increased cross sections and the smaller values (prolate-like states) leading to reduced cross sections. The bunching of the asymptotic states into the $\left\langle n_{s}-n_{z}\right\rangle$ values of $-2,-1$, 0 , and 1 is reflected in the cross sections at the largest deformations. Perhaps most striking is the lack of a correlation between the cross section and the rms radii of the associated Nilsson orbits. This radius is key in the spherical calculations, determining the extent of the nucleon wave functions with respect to the residue surface. This is not the case when the projectile is deformed. Indeed, the state with the largest rms radius at large deformations, [220]1/2, has the smallest cross section. The state is strongly prolate in shape, giving it a large

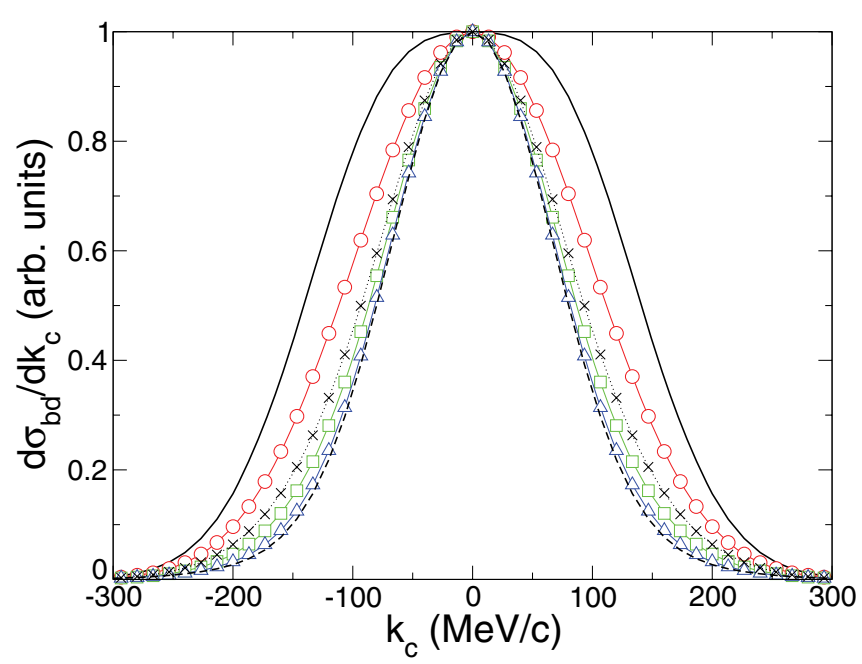

FIG. 18. (Color online) As for Fig. 9 but for the [200]1/2 Nilsson state. We also show the results obtained by weighting the spherical $1 s$ - and $0 d$-state momentum distributions by their $N=2$ model space Nilsson amplitudes for $\delta=0.2$ (black line, crosses), to be compared to the green squares. The agreement is less good than for the [211]1/2 state, shown in Fig. 16), but indicates relatively minor effects owing to the larger model space and use of the spheroidal core $S$ matrix.

$\left\langle r^{2}\right\rangle^{\frac{1}{2}}$, but with a shape that hides it more effectively within the projectile core. In essence, this is a binding energy effect. The states with the largest $n_{z}$ fall in energy (Fig. 1) precisely because their shape is well aligned with that of the deformed potential. This increases their rms radius, increases their spatial overlap with the core, but reduces their probabilities to be found near and beyond the nuclear surface, which determines the removal cross sections.

\section{DISCUSSION}

We have shown that the trends in cross sections can be understood in terms of the angular momentum compositions and the shapes of the nucleon wave functions from the Nilsson model. For prolate projectile deformations, $\delta>0$, the cross sections for prolate-like states are reduced and those for oblate-like states are increased. One can use $\left\langle n_{s}-n_{z}\right\rangle$ as a proxy for the shape of the given Nilsson state. Small admixtures generated in the larger model spaces are reflected in the calculated rms radii, but the induced changes in $\langle\ell\rangle$ and $\left\langle n_{s}-n_{z}\right\rangle$ are significantly smaller. The summed cross section for removal from all $N=2$ shell Nilsson states is approximately constant, to $\approx 5 \%$, over the range of deformations studied (from the $N=0-8$ model space plus spheroidal core calculations). Over this same range the rms radius of the core increases by $\approx 5 \%$ while the average rms radius of the Nilsson states increases by $\approx 1 \%$.

The use of a deformed core-target $S$-matrix description is critical to the changes in cross section with increases in deformation. For all states there was a marked disagreement with the $N=0-8$ space calculations that assumed a spherical core. The couplings to other major shells have an important effect on the position probability distributions of the nucleon 


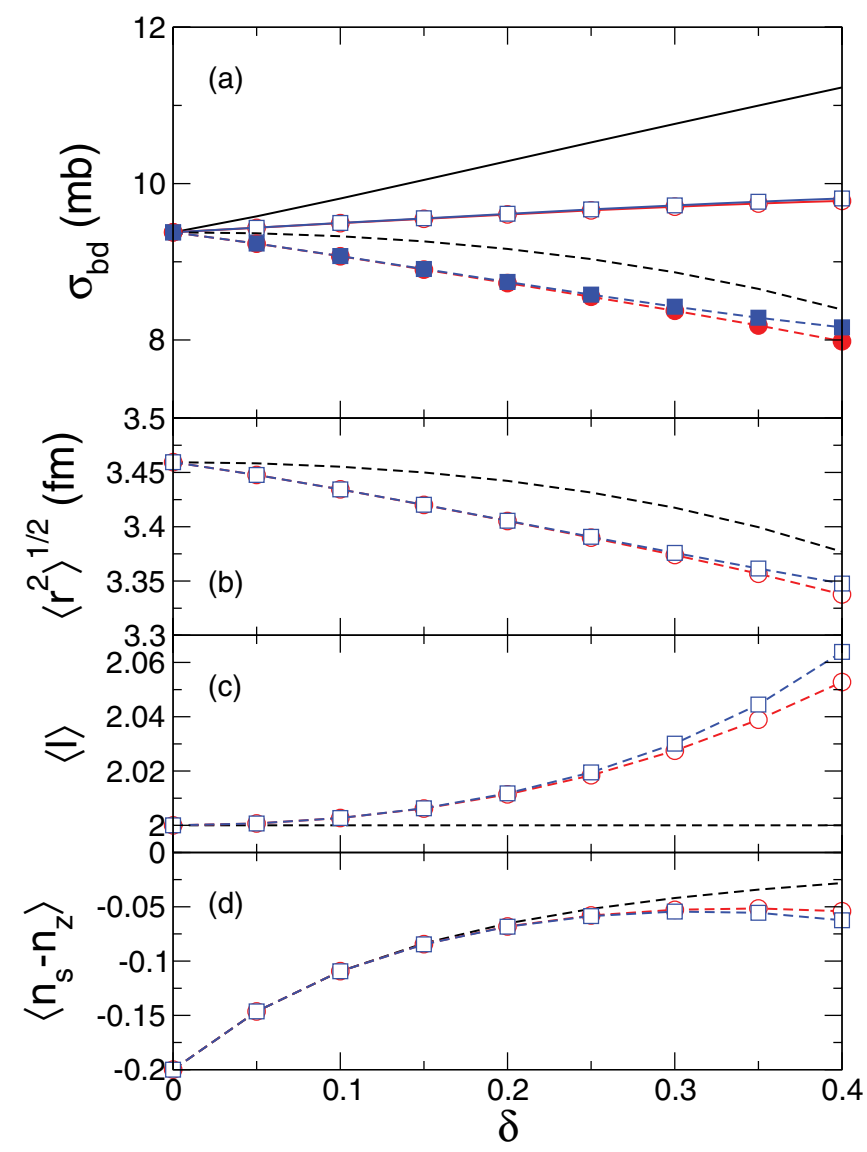

FIG. 19. (Color online) As for Fig. 7 but for the [202]3/2 Nilsson state.

wave functions relative to the projectile core, despite the relatively small changes in the $\left\langle r^{2}\right\rangle^{\frac{1}{2}},\langle\ell\rangle$, and $\left\langle n_{s}-n_{z}\right\rangle$.

Because, in the present calculations, $\delta=0$ corresponds to the conventional spherical model, the [220]1/2, [211]3/2, [202]5/2, [200]1/2, and [202]3/2 states all reproduce the $0 d$-state cross section and momentum distribution for zero deformation. The [211]1/2 state originates from the $1 s_{1 / 2}$ spherical state. Its zero deformation cross section and momentum distribution, which are larger and narrower, respectively, agree with the spherical $1 s$-state results.

From the extended basis calculations, the momentum distributions have widths characteristic of the decomposition of the Nilsson state in a spherical basis, regardless of the deformation. For the $m=1 / 2$ states dominated by $N=2$ configurations, the momentum distributions reflect the admixture of $\ell$ values, and these can be reasonably approximated by weighting the spherical $1 s$ and $0 d$ distributions by the appropriate Nilsson weights $\left[a_{v m}^{n \ell j}\right]^{2}$. For states which do not mix orbital angular momenta, the deformation dependence of the momentum distribution is very weak.

Beyond these deformation-induced changes in $\ell$ composition, deformation changes in detail the target sampling of the nucleon wave function, owing to the core-target $S$ matrix. That the calculated momentum distributions show little sensitivity to details of this sampling is not unexpected, because the observable is known to be relatively insensitive

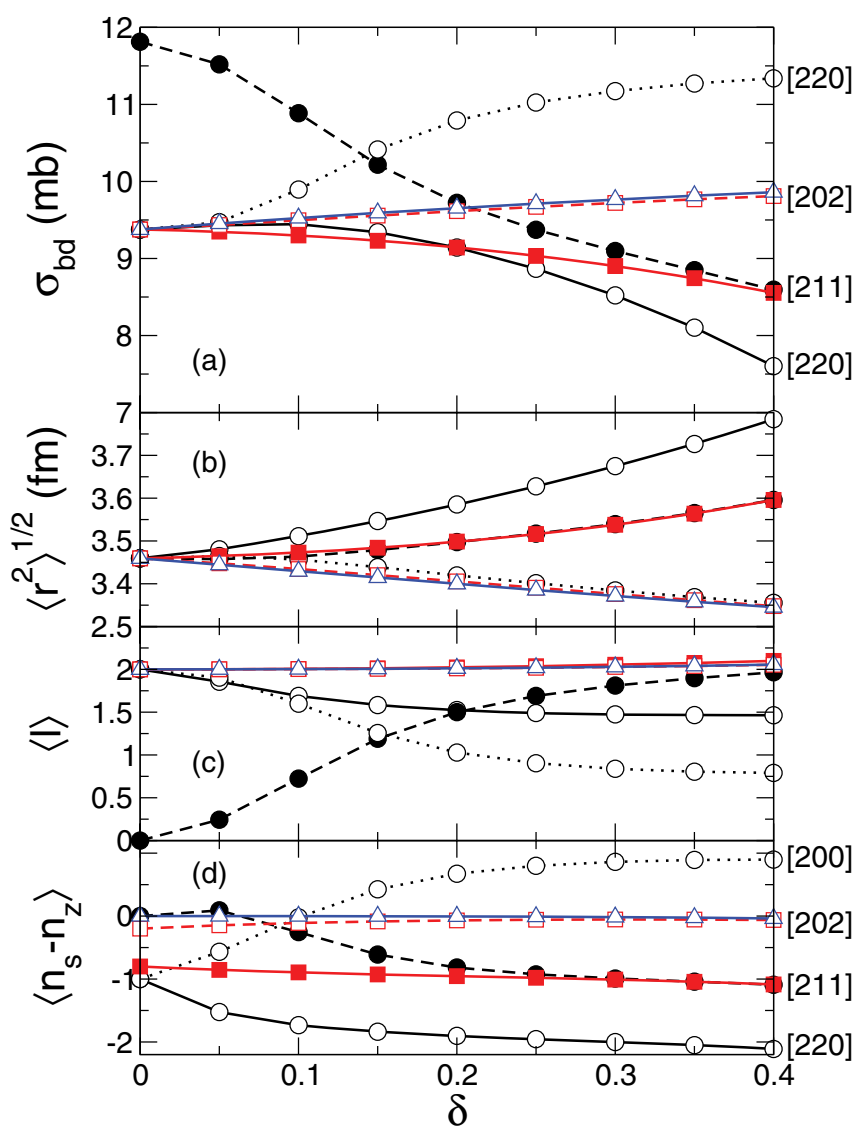

FIG. 20. (Color online) Summary of results for all $N=2$ Nilsson states calculated using a spheroidal core and the $N=0-8$ model space. The panels show (a) the cross sections, (b) the rms radii of the nucleon states, (c) $\langle\ell\rangle$, and (d) $\left\langle n_{s}-n_{z}\right\rangle$. The lines are for the [220]1/2 (black solid lines, open circles), [211]3/2 (red solid lines, solid squares), [202]5/2 (blue solid lines, open triangles), [211]1/2 (black dashed lines, solid circles), [200]1/2 (black dotted lines, open circles), and [202]3/2 (red dashed lines, open squares) states. For small $\delta$ the cross sections follow an inverse trend to $\langle\ell\rangle$. For larger $\delta$ the states split according to their asymptotic quantum numbers $\left[N n_{z} \lambda\right]$ shown on the right of the plot, the value of $\left\langle n_{s}-n_{z}\right\rangle$ determining the changes of the cross section with deformation.

to the projectile impact parameter over the ranges of interest here $[11,12]$.

We find qualitative agreement of the momentum distributions with those of Ref. [26], but there is disagreement in the detail. Whereas our zero-deformation calculations coincide with those for the appropriate spherical limit, the zero-deformation calculations presented in Ref. [26], for the $[220] 1 / 2,[211] 3 / 2$, and [202]5/2 states, which all originate from the $0 d_{5 / 2}$ state, are different (see Figs. 5 and 7-9 of Ref. [26]). We note also that in none of our calculations do we observe the development of structure (shoulders) to the residue momentum distributions, present in the results of Ref. [26], where dynamic core excitation was included. To our knowledge, such a feature has not been observed in other nucleon removal data sets on nuclei that are expected to be reasonably well deformed (e.g., Refs. [53,54]). So, it remains unclear whether such shoulders are a general feature of 
nucleon removal with deformed nuclei or are particular to the ${ }^{25} \mathrm{Al}(-1 \mathrm{p})$ reaction considered there. Further theoretical work and experiments will be required to investigate this further.

The gross changes in the spherical-basis composition of the Nilsson states should be broadly independent of the details of the model Hamiltonian parameters, $\kappa$ and $\mu$ in Eq. (1), because the states must evolve toward the asymptotic states. How rapidly this transition occurs could, in principle, be sensitive to the chosen $\kappa$ and $\mu$ values. The closer the spherical $(n \ell j)$ states are in energy at zero deformation (i.e., the smaller the values of $\kappa$ and $\mu)$, the faster the changes in the $(n \ell j)$ composition of the Nilsson states owing to the in-shell mixing will occur.

\section{SUMMARY}

We have investigated the sensitivity of fast nucleon removal cross sections and their momentum distributions to deformation of the projectile, using a strong-coupling model and using Nilsson model eigenstates for the initial bound states of the valence nucleon. We studied nucleon removal from a mass $A=28$ system, a mass region known to exhibit deformation, as a generic example from which to understand the systematics expected. The trends observed are expected to be very generally applicable to other deformed $s d$-shell systems.

We found that for small deformations the cross sections follow trends determined by the mixture of orbital angular momenta contributing to the specific Nilsson state. Thus, within the $s d$ shell, states with $m=1 / 2$ show the strongest changes. For larger (prolate) deformations the cross sections for removal of prolate-like nucleon configurations, characterized by negative values of $\left\langle n_{s}-n_{z}\right\rangle$, are suppressed. The cross sections to oblate-like configurations are enhanced. The changes in cross section over the range $\delta=0-0.4$ are as much as $20 \%$. A realistic treatment of deformation in the core-target interaction and $S$ matrix was found to be essential, as was including coupling to major shells other than $N=2$.
It was found, as for spherical models, that the residue momentum distributions reflect the orbital angular momentum content of the initial state. Despite the changes in the shape of the states, and the changes in their sampling as the core deformation changes, as reflected in the absolute cross sections, the residue longitudinal momentum distributions were found to be robustly characteristic of the orbital angular momentum decomposition of each Nilsson state. The observed effects were, naturally, largest when mixing occurs within the chosen major oscillator shell; for the $s d$-shell states of the present calculations, these are the $m=1 / 2$ states. The $m=3 / 2$ and $5 / 2$ states, dominated by $0 d$ components, have momentum distributions that changed very little with deformation.

More sophisticated models are required to make more accurate predictions of the absolute nucleon removal cross sections, including the calculation of residue final-state exclusive observables. The present calculations are limited by the sharp-cutoff approximation for the core-target $S$ matrix, estimated to reduce the calculated cross sections by $20-30 \%$. Future calculations could utilize density folding $S$ matrices [22] to overcome this shortcoming. The use of oscillator wave functions with incorrect asymptotic form leads to a further reduction of the cross section, estimated to be 5-7\%. Further, we have not considered elastic breakup of the projectile; for cases where the removed nucleon is well bound, this channel is expected to contribute to the total cross sections at the 20-25\% level. However, none of these factors are expected to alter the trends observed and the present analysis demonstrates that significant sensitivity to deformation is expected, providing the motivation for these formal and computational developments.

\section{ACKNOWLEDGMENTS}

This work was supported by the United Kingdom Science and Technology Facilities Council (STFC) under Grant No. ST/J000051/1.
[1] C. Thibault et al., Phys. Rev. C 12, 644 (1975).

[2] C. Force et al., Phys. Rev. Lett. 105, 102501 (2010).

[3] D. Santiago-Gonzalez et al., Phys. Rev. C 83, 061305(R) (2011).

[4] B. Bastin et al., Phys. Rev. Lett. 99, 022503 (2007).

[5] Z. P. Li, J. M. Yao, D. Vretenar, T. Nikšić, H. Chen, and J. Meng, Phys. Rev. C 84, 054304 (2011).

[6] A. Gade et al., Phys. Rev. C 81, 051304(R) (2010).

[7] P. Adrich et al., Phys. Rev. C 77, 054306 (2008).

[8] J. Ljungvall et al., Phys. Rev. C 81, 061301(R) (2010).

[9] W. Rother et al., Phys. Rev. Lett. 106, 022502 (2011).

[10] P. G. Hansen and J. A. Tostevin, Annu. Rev. Nucl. Part. Phys. 53, 219 (2003).

[11] P. G. Hansen, Phys. Rev. Lett. 77, 1016 (1996).

[12] H. Esbensen, Phys. Rev. C 53, 2007 (1996).

[13] D. Bazin et al., Phys. Rev. Lett. 102, 232501 (2009).

[14] K. Wimmer et al., Phys. Rev. C 85, 051603(R) (2012)

[15] A. Gade et al., Phys. Rev. Lett. 93, 042501 (2004).

[16] A. Gade et al., Phys. Rev. C 77, 044306 (2008).
[17] W. H. Dickhoff and C. Barbieri, Prog. Part. Nucl. Phys. 52, 377 (2004).

[18] O. Jensen, G. Hagen, M. Hjorth-Jensen, B. A. Brown, and A. Gade, Phys. Rev. Lett. 107, 032501 (2011).

[19] S. Ramen, C. W. Nestor, Jr., and P. Tikkanen, At. Data Nucl. Data Tables 78, 1 (2001).

[20] S. G. Nilsson, Mat. Fys. Medd. Dan. Vid. Selsk. 29(16) (1955).

[21] G. R. Satchler, Ann. Phys. 3, 275 (1958).

[22] J. A. Christley and J. A. Tostevin, Phys. Rev. C 59, 2309 (1999).

[23] G. Fäldt and R. Glauber, Phys. Rev. C 42, 395 (1990).

[24] K. Minomo, T. Sumi, M. Kimura, K. Ogata, Y. R. Shimizu, and M. Yahiro, Phys. Rev. C 84, 034602 (2011).

[25] M. Takechi et al., Phys. Lett. B 707, 357 (2012).

[26] A. Sakharuk and V. Zelevinsky, Phys. Rev. C 61, 014609 (1999).

[27] R. J. Glauber, in Lectures in Theoretical Physics, edited by W. E. Brittin, Vol. 1 (Interscience, New York, 1959), p. 315.

[28] A. Navin et al., Phys. Rev. Lett. 81, 5089 (1998).

[29] P. Batham, I. J. Thompson, and J. A. Tostevin, Phys. Rev. C 71, 064608 (2005). 
[30] A. E. Litherland, H. McManus, E. B. Paul, D. A. Bromley, and H. E. Gove, Can. J. Phys. 36, 378 (1958).

[31] H. Röpke and P. M. Endt, Nucl. Phys. A 632, 173 (1998).

[32] I. Hamamoto, Phys. Rev. C 69, 041306(R) (2004).

[33] I. Hamamoto, Phys. Rev. C 76, 054319 (2007).

[34] Y. Fujita et al., Phys. Rev. Lett. 92, 062502 (2004).

[35] B. E. Chi, Nucl. Phys. 83, 97 (1966).

[36] J. P. Davidson, Collective Models of the Nucleus (Academic Press, New York, 1968).

[37] L. E. H. Trainor, R. J. Turner, G. Tam, and L. Rosen, Can. J. Phys. 50, 740 (1972).

[38] S. G. Nilsson and I. Ragnaresson, Shapes and Shells in Nuclear Structure (Cambridge University Press, Cambridge, 1995).

[39] J. D. Talman, Nucl. Phys. A 141, 273 (1970).

[40] J. A. Tostevin, Nucl. Phys. A 682, 320c (2001).

[41] C. A. Bertulani and P. G. Hansen, Phys. Rev. C 70, 034609 (2004).
[42] D. M. Brink and G. R. Satchler, Angular Momentum (Clarendon Press, Oxford, 1993).

[43] H. Isasaki et al., Phys. Lett. B 620, 118 (2005).

[44] N. J. Stone, At. Data Nucl. Data Tables 90, 75 (2005).

[45] J. Blomqvist and A. Molinari, Nucl. Phys. A 106, 545 (1968).

[46] B. A. Brown, Phys. Rev. C 58, 220 (1998).

[47] S. K. Charagi and S. K. Gupta, Phys. Rev. C 41, 1610 (1990).

[48] W. Bauhoff et al., At. Data Nucl. Data Tables 35, 429 (1986).

[49] S. Kox et al., Phys. Rev. C 35, 1678 (1987).

[50] C. A. Bertulani and A. Gade, Comput. Phys. Commun. 175, 372 (2006).

[51] F. Barranco and E. Vigezzi, in International School of Heavy Ion Physics, edited by R. A. Broglia and P. G. Hansen (World Scientific, Singapore, 1998).

[52] M. Fukuda et al., Nucl. Phys. A 834, 461c (2010).

[53] J. R. Terry and J. L. Lecouey, Nucl. Phys. A 734, 469 (2004).

[54] J. R. Terry et al., Phys. Rev. C 77, 014316 (2008). 